\title{
VERTICES OF SPECTRAHEDRA ARISING FROM THE ELLIPTOPE, THE THETA BODY, AND THEIR RELATIVES
}

\author{
MARCEL K. DE CARLI SILVA AND LEVENT TUNÇEL
}

\begin{abstract}
Utilizing dual descriptions of the normal cone of convex optimization problems in conic form, we characterize the vertices of semidefinite representations arising from Lovász theta body, generalizations of the elliptope and related convex sets. Our results generalize vertex characterizations due to Laurent and Poljak from the 1990's. Our approach also leads us to nice characterizations of strict complementarity and to connections with some of the related literature.
\end{abstract}

\section{INTRODUCTION}

The study of the boundary structure of polyhedra arising from combinatorial optimization problems has been a very successful undertaking in the field of polyhedral combinatorics. Part of this success relies on a very rich interplay between geometric and algebraic properties of the faces of such polyhedra and corresponding combinatorial structures of the problems they encode. This remains true even in the context of some NP-hard problems, where one is generally resigned to seek partial characterizations of the boundary structure via some families of facets.

A different line of attack on combinatorial optimization problems, which has become quite popular, is that of utilizing semidefinite programming (SDP) relaxations. For the stable set problem on perfect graphs, semidefinite formulations provide the only known approach for efficient solution. Feasible regions of SDPs, known as spectrahedra, are in general much richer in complexity than polyhedra. However, or perhaps owing to that, it is reasonable to presume the existence of a wealth of combinatorial information encoded in the boundary structure of spectrahedra arising from combinatorial optimization problems. Indeed, since semidefinite optimization is a strict generalization of linear optimization, SDPs should in principle encode at least all that is known via polyhedral combinatorics.

Nonetheless, currently, results relating the boundary structure of SDPs and combinatorial properties of the corresponding problems are rather scarce. In fact, even the study of the boundary structure of SDPs per se is somewhat limited. A representative sample seems to be given by [7, 24, 23, 4, 29, 30, 3, 1, 28, 15.

A plausible reason behind this scarcity is simple to guess. From the viewpoint of linear conic optimization, a (pointed) polyhedron is the intersection of the nonnegative orthant $\mathbb{R}_{+}^{n}$ with an affine subspace of $\mathbb{R}^{n}$, whereas a spectrahedron is the intersection of the positive semidefinite cone $\mathbb{S}_{+}^{n}$ with an affine subspace of the set $\mathbb{S}^{n}$ of $n \times n$ symmetric matrices. By regarding $\mathbb{S}^{n}$ as $\mathbb{R}^{n(n+1) / 2}$ (and thus stripping off the extremely convenient algebraic structure of $\mathbb{S}^{n}$ ), one could argue that nothing is gained in terms of ambient space or affine constraints when moving from polyhedra to spectrahedra (though we shall question this very statement later on). On the other hand, the boundary structure of the cone $\mathbb{S}_{+}^{n}$, while completely understood (see, e.g., [38), is far more intricate than that of $\mathbb{R}_{+}^{n}$. The latter is in fact separable in that it may be written as the direct sum of $n$ copies of the nonnegative line $\mathbb{R}_{+}$. In this context, one is comparing the rich boundary structure of $\mathbb{S}_{+}^{n}$ with the trivial boundary structure of $\mathbb{R}_{+}$. This difference in complexity goes even further when contrasting the boundary structure of spectrahedra and polyhedra, since the intersection of an affine subspace with $\mathbb{S}_{+}^{n}$ can be so pathological that Strong Duality as well as Strict Complementarity may fail for SDPs.

In this paper, we are interested in the vertices of spectrahedra arising from combinatorial optimization problems. We focus on SDPs relaxations of the two combinatorial problems most successfully attacked via SDPs, namely, MAxCUT and the stable set problem. The aspects of the boundary structure we shall study

Research of the first author was supported in part by a Sinclair Scholarship, a Tutte Scholarship, Discovery Grants from NSERC, and by ONR research grant N00014-12-10049.

Research of the second author was supported in part by a research grant from University of Waterloo, Discovery Grants from NSERC and by ONR research grant N00014-12-10049. 
revolve around the concept of normal cone. By carefully analyzing a simple expression for the normal cone, we identify all vertices of some of the spectrahedra arising from these two problems. We also point out a simple relation between normal cones and strict complementarity, which may be helpful in proving that the latter holds for specific SDPs.

Vertices are naturally among the first objects to understand in a study of the boundary structure. Recall that a vertex of a convex set is an extreme point of that set whose normal cone is full-dimensional. For a polyhedron, extreme points and vertices coincide, and there are only finitely many of them. On the other hand, the unit ball $\left\{x \in \mathbb{R}^{d}:\|x\| \leq 1\right\}$, which is linearly isomorphic to a spectrahedron, has infinitely many extreme points and no vertices whenever $d \geq 2$. Indeed, the extreme points of the unit ball $B$ in $\mathbb{R}^{n}$ centered at the origin are precisely the unit vectors, but the normal cone at each such vector is one-dimensional. This example illustrates why an extreme point of a set whose normal cone is one-dimensional is called smooth, and how the dimension of a normal cone at a point is a measure of the "degree of non-smoothness" of the set at that point.

Vertices of a convex set can also be regarded as the only likely points to optimize a uniformly chosen linear function, in the following sense. Fix a full-dimensional convex set $\mathscr{C} \subseteq \mathbb{R}^{n}$ and a point $\bar{x} \in \mathscr{C}$. Now choose a unit vector $c \in \mathbb{R}^{n}$ uniformly at random. Then the probability that $\bar{x}$ is an optimal solution for the optimization problem $\max \{\langle c, x\rangle: x \in \mathscr{C}\}$ is positive if and only if $\bar{x}$ is a vertex of $\mathscr{C}$.

The property described above may have practical significance in some contexts where one formulates an SDP relaxation to a problem and the vertices of the feasible region correspond exactly to the combinatorial (or non-convex) objects from that problem. This kind of situation may be useful in low-rank recovery schemes; see [33. Other instances occur in combinatorial optimization, in some previous results which suggest that vertices of feasible regions of SDPs play an analogous role to that of extreme points in polyhedral combinatorics. We discuss these next.

We start with the elliptope $\mathcal{E}_{V}$, the spectrahedron arising from the famous SDP relaxation for MAxCuT utilized by Goemans and Williamson [12] in their approximation algorithm. Laurent and Poljak [20, 21] proved that all the vertices of the elliptope are rank-one, i.e., they correspond precisely to the exact solutions to the MaxCut problem. Next, we consider the theta body $\operatorname{TH}(G)$ of a graph $G$, introduced in [15] as a relaxation of the stable set polytope of $G$. Shepherd [36] observed that, by a result of [14], the vertices of $\operatorname{TH}(G)$ are precisely the incidence vectors of stable sets of $G$, i.e., again the exact solutions for the stable set problem. As far as we know, these are the only results in the literature about vertices of spectrahedra arising from combinatorial optimization problems.

One of our main results is both a generalization of the aforementioned result by Laurent and Poljak and a different version of Shepherd's observation. We describe it briefly. The theta body $\mathrm{TH}(G)$ of a graph $G=(V, E)$ is naturally described as the projection onto $\mathbb{R}^{V}$ of the feasible region of an SDP lying in the space of symmetric matrices indexed by $\{0\} \cup V$, where 0 is a new element. We denote this feasible region by $\widehat{\mathrm{TH}}(G)$. When the graph $G$ has no edges, then $\widehat{\mathrm{TH}}(G)$ is a well-known relaxation of the boolean quadric polytope and it is linearly isomorphic to the elliptope $\mathcal{E}_{\{0\} \cup V}$. We will prove that, for any graph $G$, all vertices of $\widehat{\mathrm{TH}}(G)$ are rank-one, i.e., they are the exact solutions for the stable set problem in the sense that they are the symmetric tensors of incidence vectors of stable sets in $G$.

Using similar arguments, we shall find all vertices of some well-known variants of $\widehat{\mathrm{TH}}(G)$ and $\mathcal{E}_{V}$. These include the SDP usually presented to introduce the Lovász theta number and its variants, and also the SDP studied by Kleinberg and Goemans [17] for the vertex cover problem.

We should remark that throughout the paper we only study spectrahedra in a very special form. In the literature, it is common to define spectrahedra as sets of the form $\left\{y \in \mathbb{R}^{m}: A_{0}+\sum_{i=1}^{m} y_{i} A_{i} \in \mathbb{S}_{+}^{n}\right\}$ for given matrices $A_{0}, \ldots, A_{m} \in \mathbb{S}^{n}$; the defining constraint is known as a linear matrix inequality (LMI). For the sake of convenience, we shall instead focus only on spectrahedra defined as the intersection of the cone $\mathbb{S}_{+}^{n}$ with an affine subspace of $\mathbb{S}^{n}$. An advantage is that, by confining ourselves to subsets of symmetric matrices, we retain the ability to use directly the simple but powerful algebraic structure of the underlying space $\mathbb{S}^{n}$.

We start the next section with a general set-up for convex optimization problems in conic form. In this general form, we state and prove a dual characterization of the normal cone. Then we turn to the vertices of spectrahedra arising from interesting combinatorial optimization problems. Continuing with the normal cone, duality and boundary structure themes, we conclude with a discussion of characterization of strict complementarity via the normal cone and facially exposed faces of the polar convex bodies. 


\section{Some Foundational Results}

2.1. Notation and Preliminaries. We work throughout with finite-dimensional inner-product spaces over $\mathbb{R}$, and we denote them by $\mathbb{E}$ and $\mathbb{Y}$. We denote the inner-product of $x, y \in \mathbb{E}$ by $\langle x, y\rangle$. The dual of $\mathbb{E}$ is denoted by $\mathbb{E}^{*}$. The adjoint of a linear map $\mathcal{A}: \mathbb{E} \rightarrow \mathbb{Y}$ is denoted by $\mathcal{A}^{*}$. If $\mathcal{A}$ is nonsingular, we set $\mathcal{A}^{-*}:=\left(\mathcal{A}^{*}\right)^{-1}$. If $\mathbb{K} \subseteq \mathbb{E}$ is a pointed closed convex cone with nonempty interior, the Löwner partial order on $\mathbb{E}$ is defined by setting $x \succeq_{\mathbb{K}} y$ if $x-y \in \mathbb{K}$.

Let $U, V$ be finite sets. We equip the set $\mathbb{R}^{U \times V}$ of real $U \times V$ matrices with the Frobenius inner-product defined as $\langle A, B\rangle:=\operatorname{Tr}\left(A^{\top} B\right)$, where $\operatorname{Tr}$ is the trace. Let $X \in \mathbb{R}^{U \times V}$. If $S \subseteq U$ and $T \subseteq V$, then $X[S, T]$ denotes the submatrix of $X$ in $\mathbb{R}^{S \times T}$. We also write $X[S]:=X[S, S]$.

Let $V$ be a finite set. We denote the set of $V \times V$ symmetric matrices by $\mathbb{S}^{V}$, the set of $V \times V$ positive semidefinite matrices by $\mathbb{S}_{+}^{V}$, and the set of $V \times V$ positive definite matrices by $\mathbb{S}_{++}^{V}$. For a positive integer $n$, set $[n]:=\{1, \ldots, n\}$. When $V=[n]$ we abuse the notation and write $\mathbb{S}^{n}$ for $\mathbb{S}^{[n]}$ and similarly for other sets with a superscript $V$. Note that $\mathbb{S}^{V}$ is a linear subspace of $\mathbb{R}^{V \times V}$. For $X \in \mathbb{S}^{n}$, we denote by $\lambda^{\downarrow}(X) \in \mathbb{R}^{n}$ the vector of eigenvalues of $X$ in non-increasing order. The map

$$
\text { Sym: } Y \in \mathbb{R}^{V \times V} \mapsto \frac{1}{2}\left(Y+Y^{\top}\right)
$$

is the orthogonal projection from $\mathbb{R}^{V \times V}$ onto $\mathbb{S}^{V}$. For a matrix $L \in \mathbb{R}^{V \times V}$, the map

$$
\mathcal{C}_{L}: X \in \mathbb{S}^{V} \mapsto L X L^{\top}
$$

is the congruence mapping. Note that Sym and $\mathcal{C}_{L}$ commute.

The set of nonnegative reals is denoted by $\mathbb{R}_{+}$. Let $V$ be a finite set. The standard basis vectors of $\mathbb{R}^{V}$ are $\left\{e_{i}: i \in V\right\}$. The support of $x \in \mathbb{R}^{V}$ is $\operatorname{supp}(x):=\left\{i \in V: x_{i} \neq 0\right\}$. The orthogonal group on $V$ is denoted by $\mathbb{O}(V)$. The map diag: $\mathbb{R}^{V \times V} \rightarrow \mathbb{R}^{V}$ extracts the diagonal of a matrix; its adjoint is denoted by Diag.

For a finite set $V$, we let $\left(\begin{array}{c}V \\ 2\end{array}\right)$ denote the set of all subsets of $V$ of size 2 . If $i, j \in V$ are distinct, we abbreviate $\{i, j\}$ to $i j$. We also use the Iverson bracket: if $P$ is a predicate, we set

$$
[P]:= \begin{cases}1 & \text { if } P \text { holds; } \\ 0 & \text { otherwise. }\end{cases}
$$

Let $\mathscr{C} \subseteq \mathbb{E}$ be a convex set. The relative interior of $\mathscr{C}$ is denoted by $\operatorname{ri}(\mathscr{C})$. The boundary of $\mathscr{C}$ is denoted

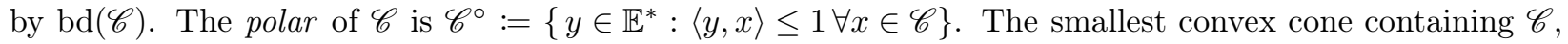
with the origin adjoined, is denoted by cone $(\mathscr{C})$. The support function of $\mathscr{C}$ is

$$
\delta^{*}(y \mid \mathscr{C}):=\sup \{\langle y, x\rangle: x \in \mathscr{C}\}
$$

and the gauge function of $\mathscr{C}$ is

$$
\gamma(x \mid \mathscr{C}):=\inf \{\lambda \geq 0: x \in \lambda \mathscr{C}\} .
$$

A convex subset $\mathscr{F}$ of $\mathscr{C}$ is a face of $\mathscr{C}$ if $x, y \in \mathscr{F}$ holds whenever $x, y \in \mathscr{C}$ are such that the open line segment between $x$ and $y$ meets $\mathscr{F}$. A face $\mathscr{F}$ of $\mathscr{C}$ is exposed if it has the form $\mathscr{F}=\mathscr{C} \cap \mathscr{H}$ for a supporting hyperplane $\mathscr{H}$ of $\mathscr{C}$.

A convex corner is a compact convex set $\mathscr{C} \subseteq \mathbb{R}_{+}^{V}$ with nonempty interior which satisfies the following property: if $0 \leq y \leq x$ and $x \in \mathscr{C}$, then $y \in \mathscr{C}$. The antiblocker of $\mathscr{C}$ is defined as

$$
\operatorname{abl}(\mathscr{C}):=\mathscr{C}^{\circ} \cap \mathbb{R}_{+}^{V} \text {. }
$$

Let $\mathscr{C} \subseteq \mathbb{E}$ be convex and let $\bar{x} \in \mathscr{C}$. Define the normal cone of $\mathscr{C}$ at $\bar{x}$ as

$$
\operatorname{Normal}(\mathscr{C} ; \bar{x}):=\left\{c \in \mathbb{E}^{*}:\langle c, x\rangle \leq\langle c, \bar{x}\rangle \forall x \in \mathscr{C}\right\} .
$$

We say that $\bar{x}$ is a vertex of $\mathscr{C}$ if $\operatorname{dim}(\operatorname{Normal}(\mathscr{C} ; \bar{x}))=\operatorname{dim}\left(\mathbb{E}^{*}\right)$.

When a convex set $\mathscr{C}$ is described as the intersection of a polyhedron and a pointed closed convex cone with nonempty interior, the Strong Duality Theorem for linear conic optimization yields a simple algebraic expression for the normal cones of $\mathscr{C}$. This may be seen as a dual characterization of normal cones.

Proposition 1. Let $\mathbb{K} \subseteq \mathbb{E}$ be a pointed closed convex cone with nonempty interior. Let $\mathcal{A}: \mathbb{E} \rightarrow \mathbb{R}^{p}$ and $\mathcal{B}: \mathbb{E} \rightarrow \mathbb{R}^{q}$ be linear functions. Let $a \in \mathbb{R}^{p}$ and $b \in \mathbb{R}^{q}$. Set $\mathscr{C}:=\{x \in \mathbb{K}: \mathcal{A}(x)=a, \mathcal{B}(x) \leq b\}$. Suppose that $\mathscr{C} \cap \operatorname{int}(\mathbb{K}) \neq \varnothing$. If $\bar{x} \in \mathscr{C}$, then

$$
\operatorname{Normal}(\mathscr{C} ; \bar{x})=\operatorname{Im}\left(\mathcal{A}^{*}\right)+\left\{\mathcal{B}^{*}(z): z \in \mathbb{R}_{+}^{q}, \operatorname{supp}(z) \cap \operatorname{supp}(\mathcal{B}(\bar{x})-b)=\varnothing\right\}-\left(\mathbb{K}^{*} \cap\{\bar{x}\}^{\perp}\right) .
$$


Proof. First we prove ' $\subseteq$ '. Let $c \in \operatorname{Normal}(\mathscr{C} ; \bar{x})$. Then $\bar{x}$ is an optimal solution for the conic programming problem

$$
\sup \{\langle c, x\rangle: \mathcal{A}(x)=a, \mathcal{B}(x) \leq b, x \in \mathbb{K}\},
$$

which has a restricted Slater point by assumption, i.e., there exists $\tilde{x} \in \operatorname{int}(\mathbb{K})$ such that $\mathcal{A}(\tilde{x})=a$ and $\mathcal{B}(\tilde{x}) \leq b$. By the Strong Duality Theorem (see, e.g., [9, Theorem 1.1]), its dual

$$
\inf \left\{\langle a, y\rangle+\langle b, z\rangle: y \in \mathbb{R}^{p}, z \in \mathbb{R}_{+}^{q}, \mathcal{A}^{*}(y)+\mathcal{B}^{*}(z) \succeq_{\mathbb{K}^{*}} c\right\}
$$

has an optimal solution $\bar{y} \oplus \bar{z} \in \mathbb{R}^{p} \oplus \mathbb{R}_{+}^{q}$ whose slack $\bar{s}:=\mathcal{A}^{*}(\bar{y})+\mathcal{B}^{*}(\bar{z})-c \in \mathbb{K}^{*}$ satisfies $\langle\bar{s}, \bar{x}\rangle=0$. (Here we use the usual inner-product $\langle a, b\rangle=a^{\top} b$ in the dual space.) By complementarity, we also have $\langle\mathcal{B}(\bar{x})-b, \bar{z}\rangle=0$. Together with $\mathcal{B}(\bar{x}) \leq b$ and $\bar{z} \in \mathbb{R}_{+}^{q}$, this implies that $\operatorname{supp}(\bar{z}) \cap \operatorname{supp}(\mathcal{B}(\bar{x})-b)=\varnothing$. Since $c=\mathcal{A}^{*}(\bar{y})+\mathcal{B}^{*}(\bar{z})-\bar{s}$, we find that $c$ lies on the set described by the RHS of (2.1).

Next we prove ' $\supseteq$ '. Let $\bar{s} \in \mathbb{K}^{*} \cap\{\bar{x}\}^{\perp}$, let $\bar{y} \in \mathbb{R}^{p}$ and $\bar{z} \in \mathbb{R}_{+}^{q}$ such that $\operatorname{supp}(\bar{z}) \cap \operatorname{supp}(\mathcal{B}(\bar{x})-b)=\varnothing$. Set $c:=\mathcal{A}^{*}(\bar{y})+\mathcal{B}^{*}(\bar{z})-\bar{s}$. If $x \in \mathscr{C}$, then

$$
\begin{aligned}
\langle c, x\rangle & =\left\langle\mathcal{A}^{*}(\bar{y}), x\right\rangle+\left\langle\mathcal{B}^{*}(\bar{z}), x\right\rangle-\langle\bar{s}, x\rangle=\langle\bar{y}, \mathcal{A}(x)\rangle+\langle\bar{z}, \mathcal{B}(x)\rangle-\langle\bar{s}, x\rangle=\langle\bar{y}, a\rangle+\langle\bar{z}, \mathcal{B}(x)\rangle-\langle\bar{s}, x\rangle \\
& \leq\langle\bar{y}, a\rangle+\langle\bar{z}, b\rangle=\langle\bar{y}, a\rangle+\langle\bar{z}, b\rangle-\langle\bar{s}, \bar{x}\rangle=\langle\bar{y}, \mathcal{A}(\bar{x})\rangle+\langle\bar{z}, \mathcal{B}(\bar{x})\rangle-\langle\bar{s}, \bar{x}\rangle \\
& =\left\langle\mathcal{A}^{*}(\bar{y}), \bar{x}\right\rangle+\left\langle\mathcal{B}^{*}(\bar{z}), \bar{x}\right\rangle-\langle\bar{s}, \bar{x}\rangle=\langle c, \bar{x}\rangle .
\end{aligned}
$$

Thus, $c \in \operatorname{Normal}(\mathscr{C} ; \bar{x})$.

Now, we move back to the special case of SDP. In this setting, it is beneficial to exploit the extra algebraic properties of the underlying space $\mathbb{S}^{n}$. A conspicuous extra feature is the fact that each point in a spectrahedron, as a matrix, has a range, a nullspace, and a rank. We shall use these concepts to massage the identity (2.1) for the normal cone and obtain a simple formula for its dimension.

We start by examining the rightmost term in (2.1), namely $\mathbb{K}^{*} \cap\{\bar{x}\}^{\perp}$, known as the conjugate face of $\bar{x}$ in $\mathbb{K}^{*}$. When $\mathbb{K}$ is the positive semidefinite cone $\mathbb{S}_{+}^{n}$, the conjugate face of a point $\bar{X}$ in $\mathbb{S}_{+}^{n}$ may be described as a lifted copy of a smaller semidefinite cone, appropriately rotated via a linear automorphism of $\mathbb{S}_{+}^{n}$ which depends only on the range of $\bar{X}$. This allows us to associate the dimension of the conjugate face to the rank of $\bar{X}$, as shown by the following well-known result (for the sake of completeness, we include a proof):

Proposition 2. Let $\bar{X} \in \mathbb{S}_{+}^{n}$. Let $Q \in \mathbb{O}(n)$ such that $X=Q \operatorname{Diag}\left(\lambda^{\downarrow}(\bar{X})\right) Q^{\top}$, and set $r:=\operatorname{rank}(\bar{X})$. Then

$$
\begin{gathered}
\mathbb{S}_{+}^{n} \cap\{\bar{X}\}^{\perp}=Q\left(0 \oplus \mathbb{S}_{+}^{n-r}\right) Q^{\top}, \\
\operatorname{ri}\left(\mathbb{S}_{+}^{n} \cap\{\bar{X}\}^{\perp}\right)=Q\left(0 \oplus \mathbb{S}_{++}^{n-r}\right) Q^{\top}, \\
\operatorname{dim}\left(\mathbb{S}_{+}^{n} \cap\{\bar{X}\}^{\perp}\right)=\left(\begin{array}{c}
\operatorname{dim}(\operatorname{Null}(\bar{X}))+1 \\
2
\end{array}\right) \\
\mathbb{S}_{+}^{n} \cap\{\bar{X}\}^{\perp}=\operatorname{cone}\left\{b b^{\top}: b \in \operatorname{Null}(\bar{X})\right\} .
\end{gathered}
$$

Proof. Set $\lambda:=\lambda^{\downarrow}(\bar{X})$. Let us prove that

$$
\mathbb{S}_{+}^{n} \cap\{\operatorname{Diag}(\lambda)\}^{\perp}=0 \oplus \mathbb{S}_{+}^{n-r} .
$$

It is clear that ' $\supseteq$ ' holds. For the reverse inclusion, let $Y \in \mathbb{S}_{+}^{n} \cap\{\operatorname{Diag}(\lambda)\}^{\perp}$. Then $0=\langle Y, \operatorname{Diag}(\lambda)\rangle=$ $\langle\operatorname{diag}(Y), \lambda\rangle$, which together with $\operatorname{diag}(Y) \geq 0$ and $\lambda \geq 0$ implies that $Y_{i i}=0$ for each $i \in \operatorname{supp}(\lambda)=[r]$. Since $Y \in \mathbb{S}_{+}^{n}$ we find that $Y_{i j}=0$ for each $i \in[r]$ and $j \in[n]$, so $Y \in 0 \oplus \mathbb{S}_{+}^{n-r}$. This proves (2.6).

Set $D:=\operatorname{Diag}(\lambda)$ and apply the map $\mathcal{C}_{Q}=\mathcal{C}_{Q}^{-*}$ to both sides of (2.6) to obtain

$$
\begin{aligned}
Q\left(0 \oplus \mathbb{S}_{+}^{n-r}\right) Q^{\top} & =\mathcal{C}_{Q}\left(\mathbb{S}_{+}^{n} \cap(\operatorname{span}\{D\})^{\perp}\right)=\mathcal{C}_{Q}\left(\mathbb{S}_{+}^{n}\right) \cap \mathcal{C}_{Q}\left((\operatorname{span}\{D\})^{\perp}\right) \\
& =\mathbb{S}_{+}^{n} \cap\left(\mathcal{C}_{Q}^{-*}(\operatorname{span}\{D\})\right)^{\perp}=\mathbb{S}_{+}^{n} \cap(\operatorname{span}\{\bar{X}\})^{\perp} .
\end{aligned}
$$

This proves (2.2).

To prove (2.3), apply ri(.) to both sides of (2.2) to get

$$
\operatorname{ri}\left(\mathbb{S}_{+}^{n} \cap\{\bar{X}\}^{\perp}\right)=\operatorname{ri}\left(\mathcal{C}_{Q}\left(0 \oplus \mathbb{S}_{+}^{n-r}\right)\right)=\mathcal{C}_{Q}\left(\operatorname{ri}\left(0 \oplus \mathbb{S}_{+}^{n-r}\right)\right)=Q\left(0 \oplus \mathbb{S}_{++}^{n-r}\right) Q^{\top} .
$$


For (2.4), use the fact that the nonsingular map $\mathcal{C}_{Q}$ preserves dimension:

$$
\begin{aligned}
\operatorname{dim}\left(\mathbb{S}_{+}^{n} \cap\{\bar{X}\}^{\perp}\right) & =\operatorname{dim}\left(\mathcal{C}_{Q}\left(0 \oplus \mathbb{S}_{+}^{n-r}\right)\right)=\operatorname{dim}\left(0 \oplus \mathbb{S}_{+}^{n-r}\right) \\
& =\operatorname{dim}\left(\mathbb{S}_{+}^{n-r}\right)=\left(\begin{array}{c}
n-r+1 \\
2
\end{array}\right)=\left(\begin{array}{c}
\operatorname{dim}(\operatorname{Null}(\bar{X}))+1 \\
2
\end{array}\right)
\end{aligned}
$$

Finally, we prove (2.5). Let $Y \in \mathbb{S}_{+}^{n}$ be arbitrary, and write $Y$ as $Y=\sum_{i=1}^{k} h_{i} h_{i}^{\top}$ where $\left\{h_{i}: i \in[k]\right\} \subseteq \mathbb{R}^{n}$. Since $Y \in \mathbb{S}_{+}^{n}$, the equation $\langle\bar{X}, Y\rangle=0$ is equivalent to $h_{i}^{\top} \bar{X} h_{i}=0$ for each $i \in[k]$. Since $\bar{X} \in \mathbb{S}_{+}^{n}$, the latter is equivalent to $h_{i} \in \operatorname{Null}(\bar{X})$ for each $i \in[k]$.

As the proof of Proposition 2 illustrates, it is often helpful to restrict our attention to a specific class of positive semidefinite matrices (e.g., diagonal matrices) for which it is easy to prove a result, and then extend it by changing the basis, e.g., by applying a congruence $\mathcal{C}_{Q}$. We now look at how normal cones behave when we apply such transformations.

Let $\mathscr{C} \subseteq \mathbb{E}$ be a convex set and let $\bar{x} \in \mathscr{C}$. If $T: \mathbb{E} \rightarrow \mathbb{Y}$ is a linear bijection, then

$$
\begin{aligned}
\operatorname{Normal}(T(\mathscr{C}) ; T(\bar{x})) & =\left\{c \in \mathbb{Y}^{*}:\langle c, T(x)\rangle \leq\langle c, T(\bar{x})\rangle \forall x \in \mathscr{C}\right\} \\
& =\left\{c \in \mathbb{Y}^{*}:\left\langle T^{*}(c), x\right\rangle \leq\left\langle T^{*}(c), \bar{x}\right\rangle \forall x \in \mathscr{C}\right\} \\
& =\left\{T^{-*}(d) \in \mathbb{Y}^{*}:\langle d, x\rangle \leq\langle d, \bar{x}\rangle \forall x \in \mathscr{C}\right\}=T^{-*}(\operatorname{Normal}(\mathscr{C} ; \bar{x})) .
\end{aligned}
$$

The identity (2.7) shows that the coordinate-free properties of normal cones remain invariant under linear bijections. In the case of SDPs, we can say a bit more in terms of the rank of a feasible matrix $\bar{X}$.

Lemma 3. Let $\mathcal{A}: \mathbb{S}^{n} \rightarrow \mathbb{R}^{p}$ and $\mathcal{B}: \mathbb{S}^{n} \rightarrow \mathbb{R}^{q}$ be linear functions. Let $a \in \mathbb{R}^{p}$ and $b \in \mathbb{R}^{q}$. Let $L \in \mathbb{R}^{n \times n}$ be nonsingular, and define

$$
\begin{gathered}
\mathcal{A}_{L}:=\mathcal{A} \circ \mathcal{C}_{L}^{-1} \quad \text { and } \quad \mathcal{B}_{L}:=\mathcal{B} \circ \mathcal{C}_{L}^{-1}, \\
\mathscr{C}:=\left\{X \in \mathbb{S}_{+}^{n}: \mathcal{A}(X)=a, \mathcal{B}(X) \leq b\right\}, \\
\mathscr{C}_{L}:=\mathcal{C}_{L}(\mathscr{C})=\left\{Y \in \mathbb{S}_{+}^{n}: \mathcal{A}_{L}(Y)=a, \mathcal{B}_{L}(Y) \leq b\right\}
\end{gathered}
$$

Then, for any $\bar{X} \in \mathscr{C}$, we have

(i) $\mathscr{C} \cap \mathbb{S}_{++}^{n} \neq \varnothing$ if and only if $\mathscr{C}_{L} \cap \mathbb{S}_{++}^{n} \neq \varnothing$;

(ii) $\operatorname{Normal}\left(\mathscr{C}_{L} ; \mathcal{C}_{L}(\bar{X})\right)=\mathcal{C}_{L}^{-*}(\operatorname{Normal}(\mathscr{C} ; \bar{X}))$ so $\operatorname{dim}\left(\operatorname{Normal}\left(\mathscr{C}_{L} ; \mathcal{C}_{L}(\bar{X})\right)\right)=\operatorname{dim}(\operatorname{Normal}(\mathscr{C} ; \bar{X}))$;

(iii) $\operatorname{Im}\left(\mathcal{A}_{L}^{*}\right)=\mathcal{C}_{L}^{-*}\left(\operatorname{Im}\left(\mathcal{A}^{*}\right)\right)$ so $\operatorname{dim}\left(\operatorname{Im}\left(\mathcal{A}_{L}^{*}\right)\right)=\operatorname{dim}\left(\operatorname{Im}\left(\mathcal{A}^{*}\right)\right)$; and analogously for $\operatorname{Im}\left(\mathcal{B}_{L}^{*}\right)$;

(iv) $\operatorname{Null}\left(\mathcal{C}_{L}(\bar{X})\right)=L^{-\mathrm{T}} \operatorname{Null}(\bar{X})$ so $\operatorname{rank}\left(\mathcal{C}_{L}(\bar{X})\right)=\operatorname{rank}(\bar{X})$.

Proof. We shall use throughout the fact that the map $\mathcal{C}_{L}$ is an automorphism of $\mathbb{S}_{+}^{n}$, and in particular it is nonsingular.

Note that $\mathscr{C}_{L} \cap \mathbb{S}_{++}^{n}=\mathcal{C}_{L}(\mathscr{C}) \cap \mathbb{S}_{++}^{n}=\mathcal{C}_{L}\left(\mathscr{C} \cap \mathbb{S}_{++}^{n}\right)$. This proves (ii). Statement (iii) follows from (2.7), whereas (iii) is elementary linear algebra. For (iv), let $h \in \mathbb{R}^{n}$ and note that $L \bar{X} L^{\top} h=0$ is equivalent to $\bar{X} L^{\top} h=0$, i.e., $L^{\top} h \in \operatorname{Null}(\bar{X})$.

2.2. Vertices of the Elliptope. We now recall some of the results from the papers [20, 21]. We first state a slightly generalized version of a result by Laurent and Poljak [21] and give a proof for the sake of completeness.

Theorem 4 ([21]). Let $\mathcal{A}: \mathbb{S}^{n} \rightarrow \mathbb{R}^{m}$ be a linear map, and let $A_{i}:=\mathcal{A}^{*}\left(e_{i}\right)$ for each $i \in[m]$. Let $b \in \mathbb{R}^{m}$ such that $\operatorname{supp}(b)=[m]$. Set $\mathscr{C}:=\left\{X \in \mathbb{S}_{+}^{n}: \mathcal{A}(X)=b\right\}$. Suppose that $\mathscr{C} \cap \mathbb{S}_{++}^{n} \neq \varnothing$ and $\operatorname{rank}\left(\sum_{i=1}^{m} A_{i}\right)=$ $\sum_{i=1}^{m} \operatorname{rank}\left(A_{i}\right)$. Then, for every $\bar{X} \in \mathscr{C}$, we have

$$
\operatorname{dim}(\operatorname{Normal}(\mathscr{C} ; \bar{X}))=\operatorname{dim}\left(\operatorname{Im}\left(\mathcal{A}^{*}\right)\right)+\left(\begin{array}{c}
\operatorname{dim}(\operatorname{Null}(\bar{X}))+1 \\
2
\end{array}\right)
$$

Proof. The proof of ' $\leq$ ' in (2.8) follows from Proposition 1 and (2.4).

Now we prove the reverse inequality. We shall use the fact that,

if $L \in \mathbb{R}^{n \times n}$ is nonsingular, then the hypotheses and conclusion of the result hold if and only if they also hold if $\mathcal{A}$ is replaced with $\mathcal{A}_{L}:=\mathcal{A} \circ \mathcal{C}_{L}^{-1}$ and $\mathscr{C}$ is replaced with $\mathscr{C}_{L}:=\mathcal{C}_{L}(\mathscr{C})$. 
Note that $\operatorname{rank}\left(\sum_{i=1}^{m} \mathcal{A}_{L}^{*}\left(e_{i}\right)\right)=\operatorname{rank}\left(\sum_{i=1}^{m} \mathcal{A}^{*}\left(e_{i}\right)\right)=\sum_{i=1}^{m} \operatorname{rank}\left(\mathcal{A}^{*}\left(e_{i}\right)\right)=\sum_{i=1}^{m} \operatorname{rank}\left(\mathcal{A}_{L}^{*}\left(e_{i}\right)\right)$ since $\operatorname{rank}\left(\mathcal{A}_{L}^{*}(x)\right)=\operatorname{rank}\left(L^{-\mathrm{T}} \mathcal{A}^{*}(x) L^{-1}\right)=\operatorname{rank}\left(\mathcal{A}^{*}(x)\right)$ for each $x \in \mathbb{R}^{m}$. Together with Lemma 3 , this proves $(2.9)$.

Let us prove that

we may assume that $A_{i} A_{j}=0$ whenever $i, j \in[m]$ are distinct.

For each $i \in[m]$, set $r_{i}:=\operatorname{rank}\left(A_{i}\right)$ and let $B_{i} \in \mathbb{R}^{n \times r_{i}}$ with full column-rank and $\operatorname{Im}\left(A_{i}\right)=\operatorname{Im}\left(B_{i}\right)$. Set $r:=\sum_{i=1}^{m} r_{i}$. Then the $n \times r$ matrix

$$
B:=\left[\begin{array}{lll}
B_{1} & \cdots & B_{m}
\end{array}\right]
$$

has full column-rank, since our hypothesis and the relation $\operatorname{Im}\left(\sum_{i=1}^{m} A_{i}\right) \subseteq \sum_{i=1}^{m} \operatorname{Im}\left(A_{i}\right)=\sum_{i=1}^{m} \operatorname{Im}\left(B_{i}\right)=$ $\operatorname{Im}(B)$ imply that

$$
r=\sum_{i=1}^{m} r_{i}=\operatorname{rank}\left(\sum_{i=1}^{m} A_{i}\right)=\operatorname{dim}\left(\operatorname{Im}\left(\sum_{i=1}^{m} A_{i}\right)\right) \leq \operatorname{dim}(\operatorname{Im}(B))=\operatorname{rank}(B) .
$$

Thus, there exists a nonsingular $L \in \mathbb{R}^{n \times n}$ such that $L B=\sum_{k=1}^{r} e_{k} e_{k}^{\top}$. If $i, j \in[m]$ are distinct, then $\operatorname{Im}\left(L B_{i}\right) \perp \operatorname{Im}\left(L B_{j}\right)$ holds and so does $\operatorname{Im}\left(\mathcal{C}_{L}\left(A_{i}\right)\right) \perp \operatorname{Im}\left(\mathcal{C}_{L}\left(A_{j}\right)\right)$. Thus, $\mathcal{C}_{L}\left(A_{i}\right) \mathcal{C}_{L}\left(A_{j}\right)=0$ whenever $i, j \in[m]$ are distinct. Thus, by replacing $\mathcal{A}$ with $\mathcal{A} \circ \mathcal{C}_{L^{-\top}}^{-1}$ and applying (2.9), this proves (2.10).

Next we shall refine (2.10) and show that we may assume that $A_{i}=\operatorname{Diag}\left(a_{i}\right)$ for each $i \in[m]$, where $a_{1}, \ldots, a_{m} \in \mathbb{R}^{n}$ are vectors with pairwise disjoint support.

Since the matrices $A_{1}, \ldots, A_{m}$ pairwise commute by (2.10), there exists $P \in \mathbb{O}(n)$ such that $P^{\top} A_{i} P$ is diagonal for each $i \in[m]$. Let $a_{i} \in \mathbb{R}^{n}$ such that $A_{i}=P \operatorname{Diag}\left(a_{i}\right) P^{\top}$ for each $i \in[m]$. For distinct $i, j \in[m]$, we have $0=P^{\top}\left(A_{i} A_{j}\right) P=\operatorname{Diag}\left(a_{i}\right) \operatorname{Diag}\left(a_{j}\right)$, whence $\operatorname{supp}\left(a_{i}\right) \cap \operatorname{supp}\left(a_{j}\right)=\varnothing$. Thus, by replacing $\mathcal{A}$ with $\mathcal{A} \circ \mathcal{C}_{P^{-1}}^{-1}$ and applying (2.9), this proves (2.11).

Let $\bar{X} \in \mathscr{C}$, let $\left\{R_{1}, \ldots, R_{p}\right\}$ be a basis of $\mathbb{S}^{n-\operatorname{rank}(\bar{X})}$, and let $Q \in \mathbb{O}(n)$ such that $\bar{X}=Q \operatorname{Diag}(\lambda) Q^{\top}$, where $\lambda:=\lambda^{\downarrow}(\bar{X})$. To prove ' $\geq$ ' in (2.8), it suffices by Proposition 1 to show that the set of matrices $\left\{A_{1}, \ldots, A_{m}\right\} \cup\left\{Q\left(0 \oplus R_{1}\right) Q^{\top}, \ldots, Q\left(0 \oplus R_{p}\right) Q^{\top}\right\}$ is linearly independent. Let $\alpha \in \mathbb{R}^{m}$ and $\beta \in \mathbb{R}^{p}$ such that

$$
\sum_{i=1}^{m} \alpha_{i} A_{i}+\sum_{j=1}^{p} \beta_{j} Q\left(0 \oplus R_{j}\right) Q^{\top}=0 .
$$

Let $u \in \operatorname{Null}(\bar{X})^{\perp}$. Then $Q^{\top} u \in Q^{\top} \operatorname{Im}(\bar{X})=\operatorname{Im}\left(Q^{\top} \bar{X}\right)=\operatorname{Im}\left(\operatorname{Diag}(\lambda) Q^{\top}\right) \subseteq \operatorname{Im}(\operatorname{Diag}(\lambda))$, whence $\operatorname{supp}\left(Q^{\top} u\right) \subseteq[\operatorname{rank}(\bar{X})]$. Thus, if we multiply (2.12) on the right by $u$ we obtain $u \in \operatorname{Null}\left(\sum_{i=1}^{m} \alpha_{i} A_{i}\right)$. So $\operatorname{Null}(\bar{X})^{\perp} \subseteq \operatorname{Null}\left(\sum_{i=1}^{m} \alpha_{i} A_{i}\right)$, or equivalently

$$
\operatorname{Im}\left(\sum_{i=1}^{m} \alpha_{i} A_{i}\right) \subseteq \operatorname{Null}(\bar{X}) .
$$

Let $k \in[m]$. Then from (2.11) we have $\operatorname{Im}\left(\alpha_{k} A_{k}\right) \subseteq \operatorname{Im}\left(\sum_{i=1}^{m} \alpha_{i} A_{i}\right) \subseteq \operatorname{Null}(\bar{X})$ so $\alpha_{k} \bar{X} A_{k}=0$. Since $0 \neq b_{k}=\left\langle A_{k}, \bar{X}\right\rangle=\operatorname{Tr}\left(A_{k} \bar{X}\right)$, we have $\bar{X} A_{k} \neq 0$, so it must be the case that $\alpha_{k}=0$. This proves that $\alpha=0$, whence $\beta=0$. This concludes the proof of (2.8).

Let $V$ be a finite set. The set

$$
\mathcal{E}_{V}:=\left\{X \in \mathbb{S}_{+}^{V}: \operatorname{diag}(X)=\bar{e}\right\},
$$

known as the elliptope, is a well-known relaxation for the cut polytope $\operatorname{conv}\left\{x x^{\top}: x \in\{ \pm 1\}^{V}\right\}$. The SDP used by Goemans and Williamson [12] in their celebrated approximation algorithm for MAxCuT has $\mathcal{E}_{V}$ as its feasible region when applied to a graph on $V$. When we apply Theorem 4 to the elliptope $\mathcal{E}_{V}$, we find that a point $\bar{X}$ of $\mathcal{E}_{V}$ is a vertex of $\mathcal{E}_{V}$ precisely when $\operatorname{dim}(\operatorname{Null}(\bar{X}))=|V|-1$ :

Corollary 5 (20]). Let $V$ be a finite set. Then a point $\bar{X}$ of $\mathcal{E}_{V}$ is a vertex of $\mathcal{E}_{V}$ if and only if $\operatorname{rank}(\bar{X})=1$. Thus, the vertices of $\mathcal{E}_{V}$ are precisely the matrices of the form $x x^{\top}$ with $x \in\{ \pm 1\}^{V}$.

In the proof of Corollary 5 by Laurent and Poljak [20, the fact that $\bar{x} \bar{x}^{\top}$ is a vertex of $\mathcal{E}_{n}$ if $\bar{x} \in\{ \pm 1\}^{n}$ follows from the simple observation that $\left\{(-1)^{\left[\bar{x}_{i} \bar{x}_{j}<0\right]} \operatorname{Sym}\left(e_{i} e_{j}^{\boldsymbol{\top}}\right): i, j \in[n]\right\} \subseteq \operatorname{Normal}\left(\mathcal{E}_{n} ; \bar{x} \bar{x}^{\boldsymbol{\top}}\right)$. For the proof that all vertices of $\mathcal{E}_{n}$ have rank one, Laurent and Poljak give the following argument, which we include for the sake of completeness: 
Proposition 6 ([20]). Let $\mathcal{A}: \mathbb{S}^{n} \rightarrow \mathbb{R}^{m}$ and $b \in \mathbb{R}^{m}$ and set $\mathscr{C}:=\left\{X \in \mathbb{S}_{+}^{n}: \mathcal{A}(X)=b\right\}$. Suppose that $\mathscr{C} \cap \mathbb{S}_{++}^{n} \neq \varnothing$. Suppose that for some $k \in[n-1]$ there exists a linearly independent subset $\left\{h_{0}\right\} \cup\left\{h_{i}: i \in[k]\right\}$ of $\mathbb{R}^{n}$ such that $\operatorname{span}\left\{\operatorname{Sym}\left(h_{0} h_{i}^{\top}\right): i \in[k]\right\} \subseteq \operatorname{Null}(\mathcal{A})$. Then every vertex of $\mathscr{C}$ has $\operatorname{rank} \leq n-k$.

Proof. We first show that,

if $L \in \mathbb{R}^{n \times n}$ is nonsingular, then the hypotheses and conclusion of the result hold if and only if they also hold if $\mathcal{A}$ is replaced with $\mathcal{A}_{L}:=\mathcal{A} \circ \mathcal{C}_{L}^{-1}$ and $\mathscr{C}$ is replaced with $\mathscr{C}_{L}:=\mathcal{C}_{L}(\mathscr{C})$.

Note that linear independence of $\left\{h_{0}\right\} \cup\left\{h_{i}: i \in[k]\right\} \subseteq \mathbb{R}^{n}$ is equivalent to that of $\left\{L h_{0}\right\} \cup\left\{L h_{i}: i \in[k]\right\}$, and the inclusion $\operatorname{span}\left\{\operatorname{Sym}\left(h_{0} h_{i}^{\top}\right): i \in[k]\right\} \subseteq \operatorname{Null}(\mathcal{A})$ is equivalent to $\operatorname{span}\left\{\operatorname{Sym}\left(L h_{0} h_{i}^{\top} L^{\top}\right): i \in[k]\right\}=$ $\mathcal{C}_{L}\left(\operatorname{span}\left\{\operatorname{Sym}\left(h_{0} h_{i}^{\top}\right): i \in[k]\right\}\right) \subseteq \operatorname{Null}\left(\mathcal{A} \circ \mathcal{C}_{L}^{-1}\right)=\operatorname{Null}\left(\mathcal{A}_{L}\right)$. The proof of (2.13) follows from these facts together with Lemma 3 .

By applying (2.13) with $L \in \mathbb{R}^{n \times n}$ nonsingular such that $L h_{0}=e_{n}$ and $L h_{i}=e_{i}$ for $i \in[k]$,

we may assume that $h_{0}=e_{n}$ and $h_{i}=e_{i}$ for all $i \in[k]$.

Set $d:=\operatorname{dim}(\operatorname{Null}(\mathcal{A}))$. Let $\operatorname{Proj}_{\operatorname{Null}(\mathcal{A})}: \mathbb{S}^{n} \rightarrow \mathbb{S}^{n}$ denote the orthogonal projection onto $\operatorname{Null}(\mathcal{A})$. Since the elements of $\left\{\operatorname{Sym}\left(e_{n} e_{i}^{\top}\right): i \in[k]\right\} \subseteq \operatorname{Null}(\mathcal{A})$ are pairwise orthogonal, we have

$$
\operatorname{Proj}_{\operatorname{Null}(\mathcal{A})}\left(\operatorname{Sym}\left(e_{n} e_{i}^{\top}\right)\right)=\operatorname{Sym}\left(e_{n} e_{i}^{\top}\right) \quad \forall i \in[k]
$$

and

there is a linear isomorphism $\varphi: \operatorname{Null}(\mathcal{A}) \rightarrow \mathbb{R}^{d}$ such that $[\varphi(X)]_{i}=X_{\text {in }}$ for all $i \in[k]$.

Let $\bar{X}$ be a vertex of $\mathscr{C}$. By Proposition 1 and (2.5), we have

$$
\operatorname{Normal}(\mathscr{C} ; \bar{X})=\operatorname{Im}\left(\mathcal{A}^{*}\right)-\left(\mathbb{S}_{+}^{n} \cap\{\bar{X}\}^{\perp}\right)=\operatorname{Im}\left(\mathcal{A}^{*}\right)-\operatorname{cone}\left\{b b^{\top}: b \in \operatorname{Null}(\bar{X})\right\} .
$$

Then

$$
\operatorname{Proj}_{\operatorname{Null}(\mathcal{A})}(\operatorname{Normal}(\mathscr{C} ; \bar{X}))=-\operatorname{cone}\left\{\operatorname{Proj}_{\operatorname{Null}(\mathcal{A})}\left(b b^{\top}\right): b \in \operatorname{Null}(\bar{X})\right\}
$$

has dimension $d$. Hence, there exists a set $\left\{b_{j}: j \in[d]\right\} \subseteq \operatorname{Null}(\bar{X})$ such that, if we define $B_{j}:=b_{j} b_{j}^{\top}$ for $j \in[d]$, the set $\left\{\operatorname{Proj}_{\operatorname{Null}(\mathcal{A})}\left(B_{j}\right): j \in[d]\right\}$ is linearly independent. So the $d \times d$ matrix $M$ whose $j$ th column is $\varphi\left(\operatorname{Proj}_{\operatorname{Null}(\mathcal{A})}\left(B_{j}\right)\right)$ is nonsingular, and its submatrix $M_{1}:=M[[k],[d]]$ has $k$ linearly independent columns. By possibly relabeling the $B_{j}$ 's, we may assume that the first $k$ columns of $M_{1}$ are linearly independent, i.e.,

$$
\left\{\left[b_{j}\right]_{n}\left(b_{j} \uparrow_{[k]}\right): j \in[k]\right\}=\left\{\varphi\left(\operatorname{Proj}_{\operatorname{Null}(\mathcal{A})}\left(B_{j}\right)\right) \uparrow_{[k]}: j \in[k]\right\} \text { is linearly independent, }
$$

where the first equation follows from (2.14) and (2.15) since $\left[\varphi\left(\operatorname{Proj}_{\mathrm{Null}(\mathcal{A})}\left(B_{j}\right)\right)\right]_{i}=\left(\operatorname{Proj}_{\mathrm{Null}(\mathcal{A})}\left(B_{j}\right)\right)_{\text {in }}=$ $\left[B_{j}\right]_{i n}=\left[b_{j}\right]_{n}\left[b_{j}\right]_{i}$ for each $i \in[k]$. In particular, $\left[b_{j}\right]_{n} \neq 0$ for each $j \in[k]$ and $\left\{b_{j}: j \in[k]\right\}$ is linearly independent. Since $b_{j} \in \operatorname{Null}(\bar{X})$ for each $j \in[k]$, we get $\operatorname{rank}(\bar{X}) \leq n-k$.

When Proposition [6 is applied to $\mathcal{E}_{n}$ in the proof of Corollary [5 with $h_{0}:=e_{n}$ and $h_{i}:=e_{i}$ for each $i \in[n-1]$, we find again that each vertex of $\mathcal{E}_{n}$ is rank-one. However, the bound provided by Proposition 6 may be quite weak: the set $\mathscr{C}:=\left\{X \in \mathbb{S}_{+}^{n}:\left\langle\operatorname{Sym}\left(e_{i} e_{j}^{\top}\right), X\right\rangle=0 \forall i j \in\left(\begin{array}{c}{[n]} \\ 2\end{array}\right)\right\}$ has a unique vertex (and extreme point) and its rank is 0 , whereas the best upper bound that Proposition 6 yields in this case is $n-1$. Still, with the same application as for the elliptope, Proposition 6 yields the following unexpected fact:

Corollary 7. Let $\mathcal{A}: \mathbb{S}^{n} \rightarrow \mathbb{R}^{m}$ be a linear map. Let $b \in \mathbb{R}^{m}$. Define $\mathscr{C}:=\left\{X \in \mathbb{S}_{+}^{n}: \mathcal{A}(X)=b\right\}$. Suppose that $\mathscr{C} \cap \mathbb{S}_{++}^{n} \neq \varnothing$. Then every vertex of $\left\{\hat{X} \in \mathbb{S}_{+}^{\{0\} \cup[n]}: \hat{X}[[n]] \in \mathscr{C}\right\}$ has rank one.

2.3. Vertices of the Theta Body. We briefly recall some basic results about the theta body of a graph and its lifted version, with emphasis on the boundary structure of the former in the setting of antiblocking duality.

Let $V$ be a finite set. Define the map

$$
\mathcal{B}_{\{0\} \cup V}: \hat{X} \in \mathbb{S}^{\{0\} \cup V} \mapsto \mathcal{B}_{\{0\}}(\hat{X}) \oplus \mathcal{B}_{V}(\hat{X}) \in \mathbb{R}^{\{0\}} \oplus \mathbb{R}^{V}
$$

where $\mathcal{B}_{\{0\}}: \mathbb{S}^{\{0\} \cup V} \rightarrow \mathbb{R}^{\{0\}}$ and $\mathcal{B}_{V}: \mathbb{S}^{\{0\} \cup V} \rightarrow \mathbb{R}^{V}$ are defined by

$$
\mathcal{B}_{\{0\}}^{*}\left(e_{0}\right):=e_{0} e_{0}^{\top} \quad \text { and } \quad \mathcal{B}_{V}^{*}\left(e_{i}\right):=\operatorname{Sym}\left(e_{i}\left(e_{i}-e_{0}\right)^{\top}\right) \quad \forall i \in V .
$$


Let $G=(V, E)$ be a graph. The lifted theta body of $G$ is defined as

$$
\widehat{\mathrm{TH}}(G):=\left\{\hat{X} \in \mathbb{S}_{+}^{\{0\} \cup V}: \mathcal{B}_{\{0\} \cup V}(\hat{X})=1 \oplus 0, \mathcal{A}_{E}(\hat{X}[V])=0\right\},
$$

where $\mathcal{A}_{E}: \mathbb{S}^{V} \rightarrow \mathbb{R}^{E}$ is defined by

$$
\mathcal{A}_{E}^{*}\left(e_{i j}\right):=\operatorname{Sym}\left(e_{i} e_{j}^{\top}\right) \quad \forall i j \in E .
$$

The theta body of $G$, first introduced in [15], is the projection

$$
\operatorname{TH}(G):=\left\{\operatorname{diag}(\hat{X}[V]) \in \mathbb{R}^{V}: \hat{X} \in \widehat{\mathrm{TH}}(G)\right\} .
$$

The facets of $\operatorname{TH}(G)$ are defined precisely by the inequalities $x_{i} \geq 0$ for each $i \in V$, and by the clique inequalities $\left\langle\mathbb{1}_{K}, x\right\rangle \leq 1$ for each clique $K$ of $G$; see, e.g., 35, Theorem 67.13]. Consequently, as mentioned by Shepherd [36], the vertices of $\operatorname{TH}(G)$ are precisely the incidence vectors of stable sets of $G$. This follows from the formula abl $(\mathrm{TH}(G))=\mathrm{TH}(\bar{G})$ (see [35, Theorem 67.12] for a proof) and the simple duality correspondence between facets and vertices in antiblocking pairs of convex corners, which may be stated as follows:

Theorem 8. Let $\mathscr{C} \subseteq \mathbb{R}_{+}^{V}$ be a convex corner. Then:

(i) the facets of $\mathscr{C}$ are determined precisely by the inequalities $x_{i} \geq 0$, for each $i \in V$, and $\langle\bar{y}, x\rangle \leq 1$, where $\bar{y}$ ranges over all vertices of $\operatorname{abl}(\mathscr{C})$ such that $\operatorname{Normal}(\mathscr{C} ; \bar{y}) \cap \mathbb{R}_{+}^{V}$ is full-dimensional.

(ii) if $x$ is a vertex of $\mathscr{C}$ with support $S$, then $x_{S}:=x \uparrow_{S}$ is a vertex of $\mathscr{C}_{S}:=\left\{z \in \mathbb{R}_{+}^{S}: z \oplus 0 \in \mathscr{C}\right\}$ and $\operatorname{Normal}\left(\mathscr{C}_{S} ; x_{S}\right) \subseteq \mathbb{R}_{+}^{S}$.

Thus, as in the case of the elliptope via Corollary 5 the vertices of the theta body $\operatorname{TH}(G)$ are the exact solutions for the problem for which $\mathrm{TH}(G)$ yields a relaxation. Our main result is the corresponding statement in matrix space, i.e., for $\widehat{\mathrm{TH}}(G)$. One may argue that the set $\mathrm{TH}(G)$, which lives in $\mathbb{R}^{V}$, is more natural to study, and indeed this is a good argument if we only consider $\operatorname{TH}(G)$ as a relaxation of the stable set polytope. However, when one actually needs to optimize a linear function over $\operatorname{TH}(G)$, the latter set is represented as a projection of $\widehat{\mathrm{TH}}(G)$, and the optimization takes place in the ambient space $\mathbb{S}\{0\} \cup V$ of $\widehat{\mathrm{TH}}(G)$. Qualitatively, $\widehat{\mathrm{TH}}(G)$ is a spectrahedron, whereas $\mathrm{TH}(G)$ is the projection of a spectrahedron. These classes of sets have quite different properties in general. We refer the reader to the paper 8 for more details.

2.4. Our Main Tool. Now we prove the principal tool for our main result: a simple algebraic expression for the dimension of the normal cone:

Theorem 9. Let $\mathcal{A}: \mathbb{S}^{n} \rightarrow \mathbb{R}^{p}$ and $\mathcal{B}: \mathbb{S}^{n} \rightarrow \mathbb{R}^{q}$ be linear functions. Let $a \in \mathbb{R}^{p}$ and $b \in \mathbb{R}^{q}$. Set $\mathscr{C}:=\left\{X \in \mathbb{S}_{+}^{n}: \mathcal{A}(X)=a, \mathcal{B}(X) \leq b\right\}$. Suppose that $\mathscr{C} \cap \mathbb{S}_{++}^{n} \neq \varnothing$. Let $\bar{X} \in \mathscr{C}$, and let $P$ denote the orthogonal projection onto $\left\{z \in \mathbb{R}^{q}: \operatorname{supp}(z) \cap \operatorname{supp}(\mathcal{B}(\bar{X})-b)=\varnothing\right\}$. Then

$$
\operatorname{dim}(\operatorname{Normal}(\mathscr{C} ; \bar{X}))=\operatorname{dim}\left(\mathbb{S}^{n}\right)-\operatorname{dim}\left(\operatorname{Null}(\mathcal{A}) \cap \operatorname{Null}(P \circ \mathcal{B}) \cap \operatorname{span}\left\{\operatorname{Sym}\left(\bar{X} u v^{\top}\right): u, v \in \mathbb{R}^{n}\right\}\right) .
$$

In particular, if $\bar{X}=\bar{x} \bar{x}^{\top}$ for some nonzero $\bar{x} \in \mathbb{R}^{n}$, then

$$
\operatorname{dim}\left(\operatorname{Normal}\left(\mathscr{C} ; \bar{x} \bar{x}^{\boldsymbol{\top}}\right)\right)=\operatorname{dim}\left(\mathbb{S}^{n}\right)-\operatorname{dim}\left(\left(\left\{A_{i} \bar{x}: i \in[p]\right\} \cup\left\{B_{i} \bar{x}: i \in[q] \backslash \operatorname{supp}\left(\mathcal{B}\left(\bar{x} \bar{x}^{\boldsymbol{\top}}\right)-b\right)\right\}\right)^{\perp}\right),
$$

where $A_{i}:=\mathcal{A}^{*}\left(e_{i}\right)$ for all $i \in[p]$ and $B_{i}:=\mathcal{B}^{*}\left(e_{i}\right)$ for all $i \in[q]$; thus, $\bar{x} \bar{x}^{\top} \in \mathscr{C}$ is a vertex of $\mathscr{C}$ if and only if $\left\{A_{i} \bar{x}: i \in[p]\right\} \cup\left\{B_{i} \bar{x}: i \in[q] \backslash \operatorname{supp}\left(\mathcal{B}\left(\bar{x} \bar{x}^{\top}\right)-b\right)\right\}$ spans $\mathbb{R}^{n}$.

Proof. We start by proving that

$$
\left[\operatorname{span}\left(\mathbb{S}_{+}^{n} \cap\{\bar{X}\}^{\perp}\right)\right]^{\perp}=\operatorname{span}\left\{\operatorname{Sym}\left(\bar{X} u v^{\top}\right): u, v \in \mathbb{R}^{n}\right\} .
$$

Let $Q \in \mathbb{O}(n)$ such that $\bar{X}=Q \operatorname{Diag}(\lambda) Q^{\top}$, where $\lambda:=\lambda^{\downarrow}(\bar{X})$. Set $D:=\operatorname{Diag}(\lambda)$ and $r:=\operatorname{rank}(\bar{X})$. Note that

$$
\left[\operatorname{span}\left(\mathbb{S}_{+}^{n} \cap\{D\}^{\perp}\right)\right]^{\perp}=\operatorname{span}\left\{\operatorname{Sym}\left(D u v^{\top}\right): u, v \in \mathbb{R}^{n}\right\}
$$


since by Proposition 2 we have

$$
\begin{aligned}
{\left[\operatorname{span}\left(\mathbb{S}_{+}^{n} \cap\{D\}^{\perp}\right)\right]^{\perp} } & =\left[\operatorname{span}\left(0 \oplus \mathbb{S}_{+}^{n-r}\right)\right]^{\perp}=\left[0 \oplus \mathbb{S}^{n-r}\right]^{\perp} \\
& =\operatorname{span}\left\{\operatorname{Sym}\left(e_{i} e_{j}^{\top}\right): i \in[r], j \in[n]\right\} \\
& =\operatorname{span}\left\{\operatorname{Sym}\left(D u v^{\top}\right): u, v \in \mathbb{R}^{n}\right\} .
\end{aligned}
$$

In the latter equality, the inclusion ' $\subseteq$ ' is obvious. For the reverse inclusion, let $u, v \in \mathbb{R}^{n}$ and note that $\operatorname{Sym}\left(D u v^{\top}\right)=\sum_{i=1}^{n} \sum_{j=1}^{n} u_{i} v_{j} \operatorname{Sym}\left(D e_{i} e_{j}^{\top}\right)=\sum_{i=1}^{r} \sum_{j=1}^{n} u_{i} v_{j} \operatorname{Sym}\left(D e_{i} e_{j}^{\top}\right)$. This proves (2.24).

To prove (2.23), apply $\mathcal{C}_{Q}$ to both sides of (2.24) to get

$$
\begin{aligned}
{\left[\operatorname{span}\left(\mathbb{S}_{+}^{n} \cap\{\bar{X}\}^{\perp}\right)\right]^{\perp} } & =\left[\operatorname{span}\left(\mathbb{S}_{+}^{n} \cap\left\{\mathcal{C}_{Q}(D)\right\}^{\perp}\right)\right]^{\perp}=\left[\operatorname{span}\left(\mathbb{S}_{+}^{n} \cap \mathcal{C}_{Q}^{-*}\left(\{D\}^{\perp}\right)\right)\right]^{\perp} \\
& =\left[\operatorname{span}\left(\mathcal{C}_{Q}^{-*}\left(\mathbb{S}_{+}^{n} \cap\{D\}^{\perp}\right)\right)\right]^{\perp}=\left[\mathcal{C}_{Q}^{-*}\left(\operatorname{span}\left(\mathbb{S}_{+}^{n} \cap\{D\}^{\perp}\right)\right)\right]^{\perp} \\
& =\mathcal{C}_{Q}\left(\left(\operatorname{span}\left(\mathbb{S}_{+}^{n} \cap\{D\}^{\perp}\right)\right)^{\perp}\right) \\
& =\mathcal{C}_{Q}\left(\operatorname{span}\left\{\operatorname{Sym}\left(D u v^{\top}\right): u, v \in \mathbb{R}^{n}\right\}\right) \\
& =\operatorname{span}\left\{\mathcal{C}_{Q}\left(\operatorname{Sym}\left(D u v^{\top}\right)\right): u, v \in \mathbb{R}^{n}\right\} \\
& =\operatorname{span}\left\{\operatorname{Sym}\left(\mathcal{C}_{Q}\left(D u v^{\top}\right)\right): u, v \in \mathbb{R}^{n}\right\} \\
& =\operatorname{span}\left\{\operatorname{Sym}\left(\bar{X} u v^{\top}\right): u, v \in \mathbb{R}^{n}\right\} .
\end{aligned}
$$

By Proposition 1 and (2.23), we have

$$
\begin{aligned}
(\operatorname{span}(\operatorname{Normal}(\mathscr{C} ; \bar{X})))^{\perp} & =\left(\operatorname{Im}\left(\mathcal{A}^{*}\right)+\operatorname{Im}\left(\mathcal{B}^{*} \circ P\right)-\operatorname{span}\left(\mathbb{S}_{+}^{n} \cap\{\bar{X}\}^{\perp}\right)\right)^{\perp} \\
& =\operatorname{Null}(\mathcal{A}) \cap \operatorname{Null}(P \circ \mathcal{B}) \cap\left[\operatorname{span}\left(\mathbb{S}_{+}^{n} \cap\{\bar{X}\}^{\perp}\right)\right]^{\perp} \\
& =\operatorname{Null}(\mathcal{A}) \cap \operatorname{Null}(P \circ \mathcal{B}) \cap \operatorname{span}\left\{\operatorname{Sym}\left(\bar{X} u v^{\top}\right): u, v \in \mathbb{R}^{n}\right\} .
\end{aligned}
$$

This proves (2.20).

For the remainder of the proof, suppose that $\bar{X}=\bar{x} \bar{x}^{\top}$ for some nonzero $\bar{x} \in \mathbb{R}^{n}$. Note that (2.23) specializes to

$$
\left[\operatorname{span}\left(\mathbb{S}_{+}^{n} \cap\left\{\bar{x} \bar{x}^{\top}\right\}^{\perp}\right)\right]^{\perp}=\left\{\operatorname{Sym}\left(\bar{x} h^{\top}\right): h \in \mathbb{R}^{n}\right\}
$$

since the RHS of (2.25) is a linear subspace of $\mathbb{S}^{n}$.

Let $h \in \mathbb{R}^{n}$. Then $\left[\mathcal{A}\left(\operatorname{Sym}\left(\bar{x} h^{\top}\right)\right)\right]_{i}=h^{\top} A_{i} \bar{x}$ for $i \in[p]$ and $\left[\mathcal{B}\left(\operatorname{Sym}\left(\bar{x} h^{\top}\right)\right)\right]_{i}=h^{\top} B_{i} \bar{x}$ for $i \in[q]$. Thus, using (2.25), we find that

$$
\begin{aligned}
\operatorname{Null}(\mathcal{A}) \cap \operatorname{Null}(P \circ \mathcal{B}) \cap & {\left[\operatorname{span}\left(\mathbb{S}_{+}^{n} \cap\{\bar{X}\}^{\perp}\right)\right]^{\perp} } \\
& =\left\{\operatorname{Sym}\left(\bar{x} h^{\top}\right): h \in\left(\left\{A_{i} \bar{x}: i \in[p]\right\} \cup\left\{B_{i} \bar{x}: i \in[q] \backslash \operatorname{supp}\left(\mathcal{B}\left(\bar{x} \bar{x}^{\top}\right)-b\right)\right\}\right)^{\perp}\right\},
\end{aligned}
$$

which has the same dimension as $\left(\left\{A_{i} \bar{x}: i \in[p]\right\} \cup\left\{B_{i} \bar{x}: i \in[q] \backslash \operatorname{supp}\left(\mathcal{B}\left(\bar{x} \bar{x}^{\top}\right)-b\right)\right\}\right)^{\perp}$ since the linear map $h \in \mathbb{R}^{n} \mapsto \operatorname{Sym}\left(\bar{x} h^{\top}\right)$ is injective. This concludes the proof of (2.21).

\section{Vertices of the Lifted Representation of the Theta Body and its Variants}

In this section, we shall use Theorem 9 to characterize the vertices of the lifted theta body, defined in (2.18). In fact, we shall identify the vertices of all the spectrahedra in a slightly more general family, which includes variations of the lifted theta body that may be used to define the graph parameters $\vartheta, \vartheta^{\prime}$ and $\vartheta^{+}$, introduced in [25, 27, 34, 37. This will allow us to determine the vertices of some other spectrahedra that arise as relaxations in combinatorial optimization; in particular, we shall see the extent to which our result generalizes the characterization of the vertices of the elliptope by Laurent and Poljak [20, 21].

Let $V$ be a finite set. Let $E \subseteq\left(\begin{array}{c}V \\ 2\end{array}\right)$. Recall the linear maps $\mathcal{B}_{\{0\} \cup V}$ and $\mathcal{A}_{E}$ defined on (2.16)-(2.19). We shall use this notation extensively throughout this section. 
We will compute all the vertices of $\widehat{\mathrm{TH}}(G)$ and its variants, which we introduce next. For a graph $G=(V, E)$, define

$$
\begin{aligned}
& \widehat{\mathrm{TH}}^{\prime}(G):=\left\{\hat{X} \in \mathbb{S}_{+}^{\{0\} \cup V}:\right.\left.\mathcal{B}_{\{0\} \cup V}(\hat{X})=1 \oplus 0, \mathcal{A}_{E}(\hat{X}[V])=0, \mathcal{A}_{\bar{E}}(\hat{X}[V]) \geq 0\right\}, \\
& \operatorname{TH}^{\prime}(G):=\left\{\operatorname{diag}(\hat{X}[V]) \in \mathbb{R}^{V}: \hat{X} \in \widehat{\mathrm{TH}}^{\prime}(G)\right\},
\end{aligned}
$$

and

$$
\begin{gathered}
\widehat{\mathrm{TH}}^{+}(G):=\left\{\hat{X} \in \mathbb{S}_{+}^{\{0\} \cup V}: \mathcal{B}_{\{0\} \cup V}(\hat{X})=1 \oplus 0, \mathcal{A}_{E}(\hat{X}[V]) \leq 0,\right\}, \\
\operatorname{TH}^{+}(G):=\left\{\operatorname{diag}(\hat{X}[V]) \in \mathbb{R}^{V}: \hat{X} \in \widehat{\mathrm{TH}}^{+}(G)\right\} .
\end{gathered}
$$

It is well known that the Lovász theta number and its variants are the support functions of these sets, i.e., for a graph $G=(V, E)$ and $w \in \mathbb{R}_{+}^{V}$, we have

$$
\begin{aligned}
\vartheta(G ; w) & =\max \{\langle w, x\rangle: x \in \mathrm{TH}(G)\}, \\
\vartheta^{\prime}(G ; w) & =\max \left\{\langle w, x\rangle: x \in \mathrm{TH}^{\prime}(G)\right\}, \\
\vartheta^{+}(G ; w) & =\max \left\{\langle w, x\rangle: x \in \mathrm{TH}^{+}(G)\right\} .
\end{aligned}
$$

We refer the reader to [18, 16] and the references therein for more details.

We may now present our main result, which identifies the vertices of $\widehat{\mathrm{TH}}(G), \widehat{\mathrm{TH}}^{\prime}(G)$, and $\widehat{\mathrm{TH}}^{+}(G)$ :

Theorem 10. Let $V$ be a finite set, and let $E^{+}, E^{-} \subseteq\left(\begin{array}{c}V \\ 2\end{array}\right)$. Set

$$
\widehat{\mathscr{C}}:=\left\{\hat{X} \in \mathbb{S}_{+}^{\{0\} \cup V}: \mathcal{B}_{\{0\} \cup V}(\hat{X})=1 \oplus 0, \mathcal{A}_{E^{+}}(\hat{X}[V]) \geq 0, \mathcal{A}_{E^{-}}(\hat{X}[V]) \leq 0\right\} .
$$

Let $\hat{X} \in \hat{\mathscr{C}}$. Then $\hat{X}$ is a vertex of $\hat{\mathscr{C}}$ if and only if $\operatorname{rank}(\hat{X})=1$.

Proof. We first prove the 'if' part. Let $\hat{X} \in \hat{\mathscr{C}}$ be rank-one, so that $\hat{X}$ is of the form $\hat{X}=(1 \oplus \bar{x})(1 \oplus \bar{x})^{\top}$ for some $\bar{x} \in \mathbb{R}^{V}$. Since $\mathcal{B}_{V}(\hat{X})=0$, we have $\bar{x} \in\{0,1\}^{V}$. Then $\left[\mathcal{B}_{\{0\}}^{*}\left(e_{0}\right)\right](1 \oplus \bar{x})=e_{0} e_{0}^{\top}(1 \oplus \bar{x})=e_{0}$ and, for $i \in V$, we have $2\left[\mathcal{B}_{V}^{*}\left(e_{i}\right)\right](1 \oplus \bar{x})=2 \operatorname{Sym}\left(e_{i}\left(e_{i}-e_{0}\right)^{\top}\right)(1 \oplus \bar{x})=\left(\bar{x}_{i}-1\right) e_{i}+\bar{x}_{i}\left(e_{i}-e_{0}\right)=\left(2 \bar{x}_{i}-1\right) e_{i}-\bar{x}_{i} e_{0}$. These vectors form a basis for $\mathbb{R}^{\{0\} \cup V}$, whence $\hat{X}$ is a vertex of $\widehat{\mathscr{C}}$ by Theorem 9 ,

Now we prove that 'only if' part. Let $\hat{X}$ be a vertex of $\widehat{\mathscr{C}}$. For each $k \in V$, define

$$
\frac{1}{2} C_{k}:=\operatorname{Sym}\left(\hat{X} e_{k} e_{0}^{\top}\right)+\sum\left\{\frac{\hat{X}_{k \ell}}{\hat{X}_{\ell \ell}} \operatorname{Sym}\left(\hat{X} e_{\ell} e_{\ell}^{\top}\right): \ell \in V, \hat{X}_{\ell \ell}>0\right\} .
$$

For $E \in\left\{E^{+}, E^{-}\right\}$, let $P_{E}$ denote the orthogonal projection onto $\left\{z \in \mathbb{R}^{E}: \operatorname{supp}(z) \cap \operatorname{supp}\left(\mathcal{A}_{E}(\hat{X}[V])\right)=\varnothing\right\}$. Let $\mathcal{F}: \mathbb{S}^{\{0\} \cup V} \rightarrow \mathbb{R}^{V} \oplus \mathbb{R}^{E+} \oplus \mathbb{R}^{E-}$ be defined as $\mathcal{F}(\hat{Y}):=\mathcal{B}_{V}(\hat{Y}) \oplus\left(P_{E^{+}} \circ \mathcal{A}_{E^{+}}(\hat{Y}[V])\right) \oplus\left(P_{E^{-}} \circ \mathcal{A}_{E^{-}}(\hat{Y}[V])\right)$ for each $\hat{Y} \in \mathbb{S}^{\{0\} \cup V}$. (Note the absence of $\{0\}$ in the index set of $\mathcal{B}_{V}$.) Let us prove that

$$
C_{k} \in \operatorname{Null}(\mathcal{F}) \text {. }
$$

Let $i, j \in\{0\} \cup V$. Then

$$
\begin{aligned}
{\left[C_{k}\right]_{i j} } & =\hat{X}_{i k}[j=0]+[i=0] \hat{X}_{k j}+\sum\left\{\frac{\hat{X}_{k \ell}}{\hat{X}_{\ell \ell}}\left(\hat{X}_{i \ell}[\ell=j]+[\ell=i] \hat{X}_{\ell j}\right): \ell \in V, \hat{X}_{\ell \ell}>0\right\} \\
& =\hat{X}_{i k}[j=0]+[i=0] \hat{X}_{k j}+\sum\left\{\frac{\hat{X}_{k \ell}}{\hat{X}_{\ell \ell}}\left(\hat{X}_{i j}[\ell=j]+[\ell=i] \hat{X}_{i j}\right): \ell \in V, \hat{X}_{\ell \ell}>0\right\} \\
& =\hat{X}_{i k}[j=0]+[i=0] \hat{X}_{k j}+\hat{X}_{i j} \sum\left\{\frac{\hat{X}_{k \ell}}{\hat{X}_{\ell \ell}}([\ell=j]+[\ell=i]): \ell \in V, \hat{X}_{\ell \ell}>0\right\} .
\end{aligned}
$$

Thus, if $i, j \in V$ are distinct and $\hat{X}_{i j}=0$, then $\left[C_{k}\right]_{i j}=0$. Let $i \in V$. Then

whereas

$$
\left[C_{k}\right]_{i i}=\hat{X}_{i i} \sum\left\{\frac{\hat{X}_{k \ell}}{\hat{X}_{\ell \ell}} 2[\ell=i]: \ell \in V, \hat{X}_{\ell \ell}>0\right\}=2\left[\hat{X}_{i i}>0\right] \hat{X}_{k i}=2 \hat{X}_{k i}
$$

$$
\left[C_{k}\right]_{i 0}=\hat{X}_{i k}+\hat{X}_{i 0} \sum\left\{\frac{\hat{X}_{k \ell}}{\hat{X}_{\ell \ell}}[\ell=i]: \ell \in V, \hat{X}_{\ell \ell}>0\right\}=\hat{X}_{i k}+\left[\hat{X}_{i i}>0\right] \hat{X}_{k i}=2 \hat{X}_{k i} .
$$


This concludes the proof of (3.1).

We claim that

$$
\text { if } k, \ell \in V \text { are such that } \hat{X}_{k k}>0 \text { and } \hat{X}_{\ell \ell}>0 \text {, then } \hat{X}_{k k}=\hat{X}_{\ell \ell}=\hat{X}_{k \ell} \text {. }
$$

Let $k, \ell \in V$ such that $\hat{X}_{k k}>0$ and $\hat{X}_{\ell \ell}>0$. Set

$$
D:=\frac{1}{\hat{X}_{k k}} C_{k}-\frac{1}{\hat{X}_{\ell \ell}} C_{\ell}
$$

Note that $\left[C_{k}\right]_{00}=2 \hat{X}_{0 k}=2 \hat{X}_{k k}$ and $\left[C_{\ell}\right]_{00}=2 \hat{X}_{0 \ell}=2 \hat{X}_{\ell \ell}$, whence $D_{00}=0$. Hence, $D \in \operatorname{Null}\left(\mathcal{B}_{\{0\}}\right)$. By (3.1), we also have $D \in \operatorname{Null}(\mathcal{F})$. Thus, by Theorem 9 , we must have $D=0$. Now from (3.3) we get

$$
0=D_{k 0}=\frac{\left[C_{k}\right]_{k 0}}{\hat{X}_{k k}}-\frac{\left[C_{\ell}\right]_{k 0}}{\hat{X}_{\ell \ell}}=\frac{2 \hat{X}_{k k}}{\hat{X}_{k k}}-\frac{2 \hat{X}_{\ell k}}{\hat{X}_{\ell \ell}} \Longrightarrow \hat{X}_{\ell \ell}=\hat{X}_{k \ell}
$$

and

$$
0=D_{\ell 0}=\frac{\left[C_{k}\right]_{\ell 0}}{\hat{X}_{k k}}-\frac{\left[C_{\ell}\right]_{\ell 0}}{\hat{X}_{\ell \ell}}=\frac{2 \hat{X}_{k \ell}}{\hat{X}_{k k}}-\frac{2 \hat{X}_{\ell \ell}}{\hat{X}_{\ell \ell}} \Longrightarrow \hat{X}_{k k}=\hat{X}_{k \ell} .
$$

This concludes the proof of (3.4).

From (3.4) we find that there exists $\eta \in \mathbb{R}$ such that

$$
\hat{X}=(1-\eta)\left[(1 \oplus 0)(1 \oplus 0)^{\top}\right]+\eta\left[\left(1 \oplus \mathbb{1}_{S}\right)\left(1 \oplus \mathbb{1}_{S}\right)^{\top}\right]
$$

where $S:=\operatorname{supp}(\operatorname{diag}(\hat{X}[V]))$. If $S=\varnothing$, the proof is complete, so assume that $S \neq \varnothing$. Then $\hat{X} \succeq 0$ is equivalent to $\eta \in[0,1]$. If $\eta=0$ the proof is complete, so assume $\eta>0$. Then (3.5) describes the extreme point $\hat{X}$ as a convex combination of two distinct points of $\hat{\mathscr{C}}$, from which we conclude that $\eta \in\{0,1\}$. Now $\operatorname{rank}(\hat{X})=1$ follows from (3.5).

We immediately obtain from Theorem 10 the vertices of all the lifted theta bodies defined above:

Corollary 11. Let $G=(V, E)$ be a graph. Let $\widehat{\mathscr{C}} \in\left\{\widehat{\mathrm{TH}}(G), \widehat{\mathrm{TH}}^{\prime}(G), \widehat{\mathrm{TH}}^{+}(G)\right\}$. Then a point $\hat{X}$ of $\widehat{\mathscr{C}}$ is a vertex of $\hat{\mathscr{C}}$ if and only if $\operatorname{rank}(\hat{X})=1$. Thus, the vertices of $\widehat{\mathscr{C}}$ are precisely the matrices of the form $\left(1 \oplus \mathbb{1}_{S}\right)\left(1 \oplus \mathbb{1}_{S}\right)^{\top}$ where $S \subseteq V$ is a stable set of $G$.

Proof. Immediate from Theorem 10, for $\widehat{\mathscr{C}}=\widehat{\mathrm{TH}}(G)$, take $E^{+}:=E^{-}:=E ;$ for $\widehat{\mathscr{C}}=\widehat{\mathrm{TH}}^{\prime}(G)$, take $E^{+}:=\left(\begin{array}{c}V \\ 2\end{array}\right)$ and $E^{-}:=E$; for $\widehat{\mathscr{C}}=\widehat{\mathrm{TH}}^{+}(G)$, take $E^{+}:=\varnothing$ and $E^{-}:=E$.

Let $V$ be a finite set. Define

$$
\begin{gathered}
\mathrm{BQ}_{\{0\} \cup V}:=\left\{\hat{X} \in \mathbb{S}_{+}^{\{0\} \cup V}: \mathcal{B}_{\{0\} \cup V}(\hat{X})=1 \oplus 0\right\}, \\
\mathrm{BQ}_{\{0\} \cup V}^{\prime}:=\left\{\hat{X} \in \mathrm{BQ}_{\{0\} \cup V}: \hat{X}[V] \geq 0\right\}, \\
\mathrm{BQ}_{\{0\} \cup V}^{\prime \prime}:=\left\{\hat{X} \in \mathrm{BQ}_{\{0\} \cup V}:\left\langle\operatorname{Sym}\left(\left(e_{0}-e_{i}\right)\left(e_{0}-e_{j}\right)^{\mathrm{T}}\right), \hat{X}\right\rangle \geq 0, \forall i j \in\left(\begin{array}{c}
V \\
2
\end{array}\right)\right\} .
\end{gathered}
$$

These sets are well-known relaxations for the boolean quadric polytope $\operatorname{conv}\left\{(1 \oplus x)(1 \oplus x)^{\top}: x \in\{0,1\}^{V}\right\}$. Also, set

$$
\text { Flip := } e_{0} e_{0}^{\top}+\sum_{i \in V} e_{i}\left(e_{0}-e_{i}\right)^{\top} \in \mathbb{R}^{(\{0\} \cup V) \times(\{0\} \cup V)} \text {. }
$$

Note that Flip $\left(1 \oplus \mathbb{1}_{S}\right)=\left(1 \oplus \mathbb{1}_{V \backslash S}\right)$ for each $S \subseteq V$. In fact, Flip is its own inverse. It is easy to check that $\mathcal{C}_{\text {Flip }}$ is an automorphism of $\mathrm{BQ}_{\{0\} \cup V}$, and that

$$
\mathcal{C}_{\text {Flip }}\left(\mathrm{BQ}_{\{0\} \cup V}^{\prime}\right)=\mathrm{BQ}_{\{0\} \cup V}^{\prime \prime} \text {. }
$$

Corollary 12. Let $V$ be a finite set. Let $\widehat{\mathscr{C}} \in\left\{\mathrm{BQ}_{\{0\} \cup V}, \mathrm{BQ}_{\{0\} \cup V}^{\prime}, \mathrm{BQ}_{\{0\} \cup V}^{\prime \prime}\right\}$. Then a point $\hat{X}$ of $\widehat{\mathscr{C}}$ is a vertex of $\widehat{\mathscr{C}}$ if and only if $\operatorname{rank}(\hat{X})=1$. Thus, the vertices of $\widehat{\mathscr{C}}$ are precisely the matrices of the form $\left(1 \oplus \mathbb{1}_{S}\right)\left(1 \oplus \mathbb{1}_{S}\right)^{\top}$ where $S \subseteq V$.

Proof. For $\widehat{\mathscr{C}} \in\left\{\mathrm{BQ}_{\{0\} \cup V}, \mathrm{BQ}_{\{0\} \cup V}^{\prime}\right\}$, this follows from Corollary11via Lemma 3 , since $\mathrm{BQ}_{\{0\} \cup V}=\widehat{\mathrm{TH}}\left(\overline{K_{V}}\right)$ and $\mathrm{BQ}_{\{0\} \cup V}^{\prime}=\widehat{\mathrm{TH}}^{\prime}\left(\overline{K_{V}}\right)$, where $K_{V}$ denotes the complete graph on $V$. For $\widehat{\mathscr{C}}=\mathrm{BQ}_{\{0\} \cup V}^{\prime \prime}$, this follows from the previous sentence together with (3.7) and Lemma 3 . 
Let $V$ be a finite set. Define

$$
\begin{aligned}
& \mathcal{E}_{\{0\} \cup V}^{\prime}:=\left\{\hat{X} \in \mathcal{E}_{\{0\} \cup V}:\left\langle\operatorname{Sym}\left(\left(e_{0}+e_{i}\right)\left(e_{0}+e_{j}\right)^{\top}\right), \hat{X}\right\rangle \geq 0, \forall i j \in\left(\begin{array}{c}
V \\
2
\end{array}\right)\right\}, \\
& \mathcal{E}_{\{0\} \cup V}^{\prime \prime}:=\left\{\hat{X} \in \mathcal{E}_{\{0\} \cup V}:\left\langle\operatorname{Sym}\left(\left(e_{0}-e_{i}\right)\left(e_{0}-e_{j}\right)^{\top}\right), \hat{X}\right\rangle \geq 0, \forall i j \in\left(\begin{array}{c}
V \\
2
\end{array}\right)\right\} .
\end{aligned}
$$

Like $\mathcal{E}_{\{0\} \cup V}$, these sets are also relaxations for the $\operatorname{conv}\left\{(1 \oplus x)(1 \oplus x)^{\top}: x \in\{ \pm 1\}^{V}\right\}$, which is a variant of the cut polytope. Also, set

$$
\text { Bool }:=\frac{1}{2} \sum_{i \in\{0\} \cup V} e_{i}\left(e_{0}+e_{i}\right)^{\top} \in \mathbb{R}^{(\{0\} \cup V) \times(\{0\} \cup V)} .
$$

Note that $\operatorname{Bool}\left(1 \oplus\left(\mathbb{1}_{S}-\mathbb{1}_{V \backslash S}\right)\right)=1 \oplus \mathbb{1}_{S}$ for each $S \subseteq V$. It is easy to check that Bool is invertible and

$$
\begin{aligned}
& \mathcal{C}_{\text {Bool }}\left(\mathcal{E}_{\{0\} \cup V}\right)=\mathrm{BQ}_{\{0\} \cup V}, \\
& \mathcal{C}_{\text {Bool }}\left(\mathcal{E}_{\{0\} \cup V}^{\prime}\right)=\mathrm{BQ}_{\{0\} \cup V}^{\prime}, \\
& \mathcal{C}_{\text {Bool }}\left(\mathcal{E}_{\{0\} \cup V}^{\prime \prime}\right)=\mathrm{BQ}_{\{0\} \cup V}^{\prime \prime} .
\end{aligned}
$$

The linear isomorphism $\mathcal{C}_{\text {Bool }}$ is quite interesting in the sense that it also maps the cut polytope to the boolean quadric polytope, the sets for which $\mathcal{E}_{\{0\} \cup V}$ and $\mathrm{BQ}_{\{0\} \cup V}$ are relaxations, respectively; see [10, 22].

Corollary 13. Let $V$ be a finite set. Let $\widehat{\mathscr{C}} \in\left\{\mathcal{E}_{\{0\} \cup V}, \mathcal{E}_{\{0\} \cup V}^{\prime}, \mathcal{E}_{\{0\} \cup V}^{\prime \prime}\right\}$. Then a point $\hat{X}$ of $\widehat{\mathscr{C}}$ is a vertex of $\widehat{\mathscr{C}}$ if and only if $\operatorname{rank}(\hat{X})=1$. Thus, the vertices of $\widehat{\mathscr{C}}$ are precisely the matrices of the form $\left(1 \oplus x_{S}\right)\left(1 \oplus x_{S}\right)^{\top}$ where $x_{S}=\mathbb{1}_{S}-\mathbb{1}_{V \backslash S}$ for some $S \subseteq V$.

Proof. Immediate from Corollary 12 and (3.8) via Lemma 3

Corollary 13 allows us to gauge the extent to which Corollary 11 generalizes Corollary 5 , the latter result characterizes the vertices for one convex set for each positive integer $n$, whereas the former does the same for all positive integers $n$ and all graphs with $n$ nodes.

Kleinberg and Goemans [17] presented SDP relaxations for the vertex cover problem. For a graph $G=$ $(V, E)$, the feasible regions of their relaxations are:

$$
\begin{gathered}
\widehat{\mathrm{VC}}(G):=\left\{\hat{X} \in \mathcal{E}_{\{0\} \cup V}:\left\langle\operatorname{Sym}\left(\left(e_{0}-e_{i}\right)\left(e_{0}-e_{j}\right)^{\top}\right), \hat{X}\right\rangle=0, \forall i j \in E\right\}, \\
\widehat{\mathrm{VC}^{\prime}}(G):=\widehat{\mathrm{VC}}(G) \cap \mathcal{E}_{\{0\} \cup V}^{\prime \prime} .
\end{gathered}
$$

Corollary 14. Let $G=(V, E)$ be a graph. Let $\widehat{\mathscr{C}} \in\left\{\widehat{\mathrm{VC}}(G), \widehat{\mathrm{VC}^{\prime}}(G)\right\}$. Then a point $\hat{X}$ of $\widehat{\mathscr{C}}$ is a vertex of $\widehat{\mathscr{C}}$ if and only if $\operatorname{rank}(\hat{X})=1$. Thus, the vertices of $\widehat{\mathscr{C}}$ are precisely the matrices of the form $\left(1 \oplus \mathbb{1}_{S}\right)\left(1 \oplus \mathbb{1}_{S}\right)^{\top}$ where $S \subseteq V$ is a vertex cover of $G$.

Proof. Immediate from Corollary [1] via Lemma 3, since we have

$$
\begin{aligned}
\widehat{\mathrm{VC}}(G) & =\left(\mathcal{C}_{\text {Bool }}^{-1} \circ \mathcal{C}_{\text {Flip }}\right)(\widehat{\mathrm{TH}}(G)), \\
\widehat{\mathrm{VC}}^{\prime}(G) & =\left(\mathcal{C}_{\text {Bool }}^{-1} \circ \mathcal{C}_{\text {Flip }}\right)\left(\widehat{\mathrm{TH}}^{\prime}(G)\right) .
\end{aligned}
$$

The Lovász theta number is sometimes presented using another SDP, in a smaller dimensional space. We shall now show that the vertices of the feasible region of this SDP do not coincide with what would be considered its exact solutions:

Theorem 15. Let $V$ be a finite set, and let $E^{+}, E^{-} \subseteq\left(\begin{array}{l}V \\ 2\end{array}\right)$. Set

$$
\mathscr{C}:=\left\{X \in \mathbb{S}_{+}^{V}: \operatorname{Tr}(X)=1, \mathcal{A}_{E^{+}}(X) \geq 0, \mathcal{A}_{E^{-}}(X) \leq 0\right\},
$$

$H:=\left(V, E^{+1} \cup E^{-1}\right)$, and $n:=|V|$. Then the set of vertices of $\mathscr{C}$ is $\left\{e_{k} e_{k}^{\top}: \operatorname{deg}_{H}(k)=n-1\right\}$.

Proof. We first show that

$$
\text { if } \bar{X} \text { is a vertex of } \mathscr{C} \text {, then } \bar{X}=e_{k} e_{k}^{\top} \text { for some } k \in V \text {. }
$$

Let $\bar{X}$ be a vertex of $\mathscr{C}$. Let $k, \ell \in V$ be distinct. Set

$$
\frac{1}{2} D:=\bar{X}_{\ell \ell} \operatorname{Sym}\left(\bar{X} e_{k} e_{k}^{\top}\right)-\bar{X}_{k k} \operatorname{Sym}\left(\bar{X} e_{\ell} e_{\ell}^{\top}\right) .
$$


If $i, j \in V$, then

$$
\begin{aligned}
D_{i j} & =\bar{X}_{\ell \ell}\left(\bar{X}_{i k}[k=j]+[k=i] \bar{X}_{k j}\right)-\bar{X}_{k k}\left(\bar{X}_{i \ell}[\ell=j]+[\ell=i] \bar{X}_{\ell j}\right) \\
& =\bar{X}_{\ell \ell} \bar{X}_{i j}([k=j]+[k=i])-\bar{X}_{k k} \bar{X}_{i j}([\ell=j]+[\ell=i]) \\
& =\bar{X}_{i j}\left[\bar{X}_{\ell \ell}([k=j]+[k=i])-\bar{X}_{k k}([\ell=j]+[\ell=i])\right] .
\end{aligned}
$$

For $i j \in\left(\begin{array}{c}V \\ 2\end{array}\right)$, we clearly have $D_{i j}=0$ whenever $\bar{X}_{i j}=0$. We also have

$$
\operatorname{Tr}(D)=D_{k k}+D_{\ell \ell}=\left(2 \bar{X}_{k k} \bar{X}_{\ell \ell}\right)+\left(-2 \bar{X}_{\ell \ell} \bar{X}_{k k}\right)=0 .
$$

Note that $|V|^{-1} I$ lies in $\mathscr{C} \cap \mathbb{S}_{++}^{V}$, so we may apply Theorem 9 to get $D=0$. Thus, $0=D_{k k}=2 \bar{X}_{k k} \bar{X}_{\ell \ell}$. Since $k$ and $\ell$ were arbitrary, (3.9) follows from $\operatorname{Tr}(\bar{X})=1$.

We will now show that,

$$
\text { for } k \in V \text {, the point } e_{k} e_{k}^{\top} \text { is a vertex of } \mathscr{C} \text { if and only if } \operatorname{deg}_{H}(k)=n-1 \text {. }
$$

Let $k \in V$. Set $E:=E^{+} \cup E^{-}$. By Theorem 9, the point $e_{k} e_{k}^{\top}$ is a vertex of $\mathscr{C}$ if and only if $\left\{e_{k}\right\} \cup$ $\left\{\operatorname{Sym}\left(e_{i} e_{j}^{\top}\right) e_{k}: i j \in E\right\}$ spans $\mathbb{R}^{V}$. The latter set is $\left\{e_{k}\right\} \cup\left\{[j=k] e_{i}+[i=k] e_{j}: i j \in E\right\}=\left\{e_{k}\right\} \cup\left\{e_{j}: j k \in E\right\}$, so it spans $\mathbb{R}^{V}$ precisely when $\operatorname{deg}_{H}(k)=n-1$.

The result now follows from (3.9) and (3.10).

Corollary 16. Let $G=(V, E)$ be a graph. Set $P:=\left\{k \in V: \operatorname{deg}_{G}(k)=|V|-1\right\}$. Then

(i) the set of vertices of $\left\{X \in \mathbb{S}_{+}^{V}: \operatorname{Tr}(X)=1, \mathcal{A}_{E}(X)=0\right\}$ is $\left\{e_{k} e_{k}^{\top}: k \in P\right\}$;

(ii) the set of vertices of $\left\{X \in \mathbb{S}_{+}^{V}: \operatorname{Tr}(X)=1, \mathcal{A}_{E}(X)=0, \mathcal{A}_{\bar{E}}(X) \geq 0\right\}$ is $\left\{e_{k} e_{k}^{\top}: k \in V\right\}$;

(iii) the set of vertices of $\left\{X \in \mathbb{S}_{+}^{V}: \operatorname{Tr}(X)=1, \mathcal{A}_{E}(X) \leq 0\right\}$ is $\left\{e_{k} e_{k}^{\top}: k \in P\right\}$.

Proof. Immediate from Theorem [15, as in the proof of Corollary 11$]$

The results in this section significantly extend the combinatorially-inspired spectrahedra whose vertices are completely understood. However, we do not know the set of vertices of some of their simplest variants, such as $\mathrm{BQ}_{\{0\} \cup V}^{\prime} \cap \mathrm{BQ}_{\{0\} \cup V}^{\prime \prime}$ or even

$$
\left\{\hat{X} \in \mathrm{BQ}_{\{0\} \cup V}:\left\langle\operatorname{Sym}\left(e_{i}\left(e_{i}-e_{j}\right)^{\mathrm{\top}}\right), \hat{X}\right\rangle \geq 0, \forall(i, j) \in V \times V\right\} ;
$$

the constraints of the latter usually appear in spectrahedra arising from the lift-and-project operator of Lovász and Schrijver [26]. This is just a hint of the complexity of the vertex structure of spectrahedra that we warned about in the introduction. We roughly discuss some other difficulties next.

When considering sufficient conditions which bound the rank of vertices of a spectrahedron, such as the ones from Theorem 4 and Proposition [6] ideally one seeks to obtain coordinate-free conditions that are easy to check and that have a built-in detection for a change of basis. Let us use Theorem 4 to explain this. Suppose we replace the rank hypothesis from that theorem with the condition that $A_{i} A_{j}=0$ for distinct $i, j \in[n]$. Note that we eventually reach this assumption in (2.10) in its proof. Then the modified theorem would be applicable to the elliptope $\mathcal{E}_{\{0\} \cup V}$, but not to its linear isomorphic image

$$
\left\{\hat{X} \in \mathbb{S}_{+}^{\{0\} \cup V}:\left\langle\left(e_{0}-2 e_{i}\right)\left(e_{0}-2 e_{i}\right)^{\top}, \hat{X}\right\rangle=1 \forall i \in\{0\} \cup V\right\},
$$

which is nothing but $\mathrm{BQ}_{\{0\} \cup V}$. What happened in this case was that we have the following equivalence: there exists a nonsingular $L \in \mathbb{R}^{n \times n}$ such that $\mathcal{C}_{L}\left(A_{i}\right) \mathcal{C}_{L}\left(A_{j}\right)=0$ for distinct $i, j \in[n]$ if and only if the rank condition from Theorem 4 holds. That is, a simple algebraic condition subsumes an existential predicate about a convenient basis; the rank condition factors out the trivial congruences. This is in contrast with the existential hypothesis from Proposition 6, which is harder to check, and thus harder to apply. However, Theorem 4 is not yet entirely coordinate-free; this may be seen from the fact that it does not apply directly to $\mathrm{BQ}_{\{0\} \cup V}$ using its description in (3.6a) , since the theorem requires the RHS of the defining linear equations to be nonzero everywhere. In this sense, Theorem 4 still has some room for improvement.

The algebraic aspects just described have a complementary role to geometry in some situations. For instance, it is easy to see how to start with a spectrahedron all of whose vertices have rank one and transform it into one that has all vertices of rank two; one could take a direct sum with a constant nonzero block, and apply a congruence transformation to "hide" the triviality of this transformation. Here the geometric aspect 
of the transformation is trivial. However, a broad sufficient condition to bound the rank of vertices needs to factor out all these congruences. This seems hard to describe algebraically without an existential hypothesis. On the other direction, Corollary 7 describes a transformation of spectrahedra that is trivial in terms of algebra, but geometrically it modifies the boundary structure drastically.

The above results indicate that the approach presented here and in the previous literature we cited, may lead to further fruitful results. To indicate some of this potential, we move to some other aspects of the boundary structure beyond the vertices, but continue to utilize the characterizations of normal cone and duality themes.

\section{Strict Complementarity}

We continue considering the consequences of the dual characterization (2.1) of the normal cone at a boundary point which has been very fruitful so far. Note that Proposition 1, which is a dual characterization of the normal cone at $\bar{x}$, shows explicitly that the normal cone at $\bar{x}$ of the feasible region of a conic optimization problem is the Minkowski sum of a polyhedral cone (defined by $\mathcal{A}, \mathcal{B}$, and $\bar{x}$ ) and the conjugate of the minimal face of $\mathbb{K} \subseteq \mathbb{E}$ containing $\bar{x}$. By taking the relative interior of both sides of this characterization, we shall find a strong connection to strict complementarity. We first recall a definition by Pataki [29].

Definition 17. Let $\mathbb{K} \subseteq \mathbb{E}$ be a pointed closed convex cone with nonempty interior. Let $\mathcal{A}: \mathbb{E} \rightarrow \mathbb{Y}^{*}$ be a linear function. Let $c \in \mathbb{E}^{*}$ and $b \in \mathbb{Y}^{*}$. Set

$$
\begin{gathered}
\mathscr{C}_{P}:=\{x \in \mathbb{K}: \mathcal{A}(x)=b\}, \\
\mathscr{C}_{D}:=\left\{s \in \mathbb{K}^{*}: s \in \operatorname{Im}\left(\mathcal{A}^{*}\right)-c\right\} .
\end{gathered}
$$

We say that a pair $(\bar{x}, \bar{s}) \in \mathscr{C}_{P} \times \mathscr{C}_{D}$ is strictly complementary if there exists a face $F$ of $\mathbb{K}$ such that

$$
\bar{x} \in \operatorname{ri}(F) \text { and } \bar{s} \in \operatorname{ri}\left(F^{\triangle}\right) \text {. }
$$

In the above $F^{\triangle}:=\mathbb{K}^{*} \cap F^{\perp}$ is the conjugate face of $F$. Note that the condition $\bar{x} \in \operatorname{ri}(F)$ for a face $F$ of $\mathbb{K}$ is equivalent to the fact that $F$ is the smallest face of $\mathbb{K}$ that contains $\bar{x}$; see, e.g., [32, Theorem 18.1]. Thus, if we define $F$ as the smallest face of $\mathbb{K}$ containing $\bar{x}$, then $F^{\triangle}=\mathbb{K}^{*} \cap\{\bar{x}\}^{\perp}$. With this in mind, the next observation becomes clear from Proposition 1 (it appears in a slightly different form in [6, Sec. 2]):

Proposition 18. In the context of Definition 17 suppose that $\mathscr{C}_{P} \cap \operatorname{int}(\mathbb{K}) \neq \varnothing$. Let $\bar{x} \in \mathscr{C}_{P}$. Then there exists $\bar{s} \in \mathscr{C}_{D}$ such that $(\bar{x}, \bar{s})$ is strictly complementary if and only if $c \in \operatorname{ri}\left(\operatorname{Normal}\left(\mathscr{C}_{P} ; \bar{x}\right)\right)$.

Proof. The condition for strict complementarity of a pair $(\bar{x}, \bar{s})$ requires a face $F$ of $\mathbb{K}$ to satisfy $\bar{x} \in \operatorname{ri}(F)$. Since our primal feasible $\bar{x}$ is fixed, the face $F$ is also fixed to be the minimal face of $\mathbb{K}$ containing $\bar{x}$. Thus, given $\bar{s} \in \mathscr{C}_{D}$, strict complementarity of $(\bar{x}, \bar{s})$ is equivalent to the membership $\bar{s} \in \operatorname{ri}\left(F^{\triangle}\right)=\operatorname{ri}\left(\mathbb{K}^{*} \cap\{\bar{x}\}^{\perp}\right)$. Under the assumption that $\mathscr{C}_{P} \cap \operatorname{int} \mathbb{K} \neq \varnothing$, we have by Proposition 1 that

$$
\operatorname{ri}\left(\operatorname{Normal}\left(\mathscr{C}_{P} ; \bar{x}\right)\right)=\operatorname{Im}\left(\mathcal{A}^{*}\right)-\operatorname{ri}\left(\mathbb{K}^{*} \cap\{\bar{x}\}^{\perp}\right) .
$$

Suppose that $\bar{s} \in \mathscr{C}_{D}$ is such that $\bar{s} \in \operatorname{ri}\left(\mathbb{K}^{*} \cap\{\bar{x}\}^{\perp}\right)$. Since $\bar{s} \in \mathscr{C}_{D}$, we have $c \in \operatorname{Im}\left(\mathcal{A}^{*}\right)-\bar{s} \subseteq$ $\operatorname{Im}\left(\mathcal{A}^{*}\right)-\operatorname{ri}\left(\mathbb{K}^{*} \cap\{\bar{x}\}^{\perp}\right)=\operatorname{ri}\left(\operatorname{Normal}\left(\mathscr{C}_{P} ; \bar{x}\right)\right)$ by (4.2). For the converse, suppose that $c \in \operatorname{ri}\left(\operatorname{Normal}\left(\mathscr{C}_{P} ; \bar{x}\right)\right)$. Then by (4.2) there exists $\bar{s} \in \operatorname{ri}\left(\mathbb{K}^{*} \cap\{\bar{x}\}^{\perp}\right)$ such that $c \in \operatorname{Im}\left(\mathcal{A}^{*}\right)-\bar{s}$. In particular, $\bar{s} \in \mathscr{C}_{D}$. Thus, $(\bar{x}, \bar{s})$ is strictly complementary.

The above proposition already implies that strict complementarity is locally generic in the following sense:

Corollary 19. In the context of Definition 17, suppose that $\mathscr{C}_{P} \cap \operatorname{int}(\mathbb{K}) \neq \varnothing$. Let $\bar{x} \in \mathscr{C}_{P}$. Consider the set $\operatorname{Normal}\left(\mathscr{C}_{P} ; \bar{x}\right)$ of all $c \in \mathbb{E}^{*}$ for which $\bar{x}$ is optimal for $\sup \left\{\langle c, x\rangle: x \in \mathscr{C}_{P}\right\}$. Set $d:=\operatorname{dim}\left(\operatorname{Normal}\left(\mathscr{C}_{P} ; \bar{x}\right)\right)$. Then, the set

$$
\left\{c \in \operatorname{Normal}\left(\mathscr{C}_{P} ; \bar{x}\right): \text { there does not exist } \bar{s} \in \mathscr{C}_{D} \text { such that }(\bar{x}, \bar{s}) \text { is strictly complementary }\right\}
$$

is of measure zero with respect to $d$-dimensional Hausdorff measure.

Proof. Normal cone is closed and convex, and we may assume that it is pointed. Then, we can analyze its boundary structure by taking a cross-section of it via intersection by a hyperplane whose normal is defined by an interior point of the cone dual to the normal cone (in the $d$-dimensional affine span of the cone). Let $\mathscr{C}$ denote this cross-section (whose dimension is $d-1$ ). It is well-known that the set of all $(d-2)$-dimensional 
faces of such a convex set $\mathscr{C}$ is a countable set and furthermore the union of the relative boundaries of these faces have zero $(d-2)$-dimensional Hausdorff measure (a result of Larman [19]). Therefore, for the $(d-1)$-dimensional convex set $\mathscr{C}$, its boundary has a zero $(d-1)$-dimensional Hausdorff measure. Hence, the relative boundary of the normal cone in consideration is of measure zero with respect to $d$-dimensional Hausdorff measure. Therefore, the claim follows from Proposition 18 .

There are similar strict complementarity results in the literature starting with Alizadeh, Haeberly and Overton [2], Pataki and the second author [31], Gortler and Thurston [13], Nie, Ranestad and Sturmfels [28, and Drusvyatskiy and Lewis [11. All of these results are generic. Many of these papers also address various related notions of nondegeneracy and establish that it too is generic. However, it is well-known in LP literature that degeneracy arises often in applications and in many cases "naturally." Therefore, it is of interest to characterize when a certain geometric/algebraic condition can guarantee nondegeneracy or strict complementarity.

We shall next present a characterization of strict complementarity which may be helpful in proving that some specific SDPs satisfy strict complementarity. First we recall an elementary result in convex analysis (for the sake of completeness a proof is included in the appendix):

Proposition 20. Let $\mathscr{C} \subseteq \mathbb{E}$ be a compact convex set. Then the gauge function $\gamma(\cdot \mid \mathscr{C})$ of $\mathscr{C}$ is closed.

Using Proposition 20, we slightly extend a characterization of the exposed faces of the polar from [6] (again, for the sake of completeness, a proof is included in the appendix):

Theorem 21. Let $\mathscr{C} \subseteq \mathbb{E}$ be a compact convex set. Then the nonempty exposed faces of $\mathscr{C}^{\circ}$ other than $\mathscr{C}^{\circ}$ itself are precisely the nonempty sets of the form

$$
\mathscr{F}_{\bar{x}}:=\{y \in \operatorname{Normal}(\mathscr{C} ; \bar{x}):\langle y, \bar{x}\rangle=1\}
$$

as $\bar{x}$ ranges over $\mathscr{C}$. Moreover, for any such face,

$$
\operatorname{ri}\left(\mathscr{F}_{\bar{x}}\right)=\{y \in \operatorname{ri}(\operatorname{Normal}(\mathscr{C} ; \bar{x})):\langle y, \bar{x}\rangle=1\} .
$$

Now we can characterize exactly the existence of strictly complementary solutions for linear programs in conic form for a rich class of objective functions.

Theorem 22. Let $\mathbb{K} \subseteq \mathbb{E}$ be a pointed closed convex cone with nonempty interior. Let $\mathcal{A}: \mathbb{E} \rightarrow \mathbb{Y}^{*}$ be a linear function, and let $b \in \mathbb{Y}^{*}$. Set $\mathscr{C}:=\{x \in \mathbb{K}: \mathcal{A}(x)=b\}$. Suppose that $\mathscr{C} \cap \operatorname{int}(\mathbb{K}) \neq \varnothing$ and that $\mathscr{C}$ is compact. Then the following are equivalent:

(i) for every $c \in \mathbb{E}^{*} \backslash[\operatorname{cone}(\mathscr{C})]^{\circ}$, the optimization problem $\max \{\langle c, x\rangle: \mathcal{A}(x)=b, x \in \mathbb{K}\}$ and its dual have a strictly complementary pair of optimal solutions;

(ii) $\mathscr{C}^{\circ}$ is facially exposed.

Proof. We start with the forward implication. Let $\mathscr{F}$ be a face of $\mathscr{C}^{\circ}$ such that $\varnothing \neq \mathscr{F} \neq \mathscr{C}^{\circ}$. Let $c \in \operatorname{ri}(\mathscr{F})$. Since $\mathscr{C}$ is compact, $\mathscr{C}^{\circ}$ has nonempty interior. Now $\mathscr{F} \neq \mathscr{C}^{\circ}$ implies that $c \in \mathscr{F} \subseteq \operatorname{bd}\left(\mathscr{C}^{\circ}\right)$. Hence, $c$ lies in some exposed face of $\mathscr{C}^{\circ}$. Thus, by Theorem 21], there is some $\bar{x} \in \mathscr{C}$ such that $c \in \mathscr{F}_{\bar{x}}$, using the notation from (4.3). Thus, $\langle c, \bar{x}\rangle=1$, which shows that $c \notin[\operatorname{cone}(\mathscr{C})]^{\circ}$. By hypothesis, $\max \{\langle c, x\rangle: \mathcal{A}(x)=b, x \in \mathbb{K}\}$ and its dual have a strictly complementary pair of optimal solutions, so that $c \in \operatorname{ri}(\operatorname{Normal}(\mathscr{C} ; \hat{x}))$ for some $\hat{x} \in \mathscr{C}$ by Proposition[18, Note that $1=\langle c, \bar{x}\rangle \leq\langle c, \hat{x}\rangle$ and $c \in \mathscr{C}^{\circ}$ so $\langle c, \hat{x}\rangle=1$. Thus, we find by Theorem 21] that $c \in \operatorname{ri}\left(\mathscr{F}_{\hat{x}}\right)$. But this means that $\mathscr{F}=\mathscr{F}_{\hat{x}}$, so that $\mathscr{F}$ is exposed.

Suppose next that $\mathscr{C}^{\circ}$ is facially exposed, and let $c \in \mathbb{E}^{*} \backslash[\operatorname{cone}(\mathscr{C})]^{\circ}$. Let $\bar{x} \in \arg \max _{x \in \mathscr{C}}\langle c, x\rangle$. Note that $\langle c, \bar{x}\rangle \leq 0$ would imply that $c \in[\operatorname{cone}(\mathscr{C})]^{\circ}$, so $\langle c, \bar{x}\rangle>0$. Set $\bar{c}:=c /\langle c, \bar{x}\rangle$ so that $\langle\bar{c}, \bar{x}\rangle=1$. Together with $\bar{c} \in \operatorname{Normal}(\mathscr{C} ; \bar{x})$, this implies that $\bar{c} \in \mathscr{F}_{\bar{x}}$, using the notation from (4.3). By Theorem 21] it follows that $\bar{c}$ lies in $\operatorname{bd}\left(\mathscr{C}^{\circ}\right)$. Since $\mathscr{C}^{\circ}$ is facially exposed, there exists an exposed face $\mathscr{F}$ of $\mathscr{C}^{\circ}$ such that $\bar{c} \in \operatorname{ri}(\mathscr{F})$. By Theorem 21, there exists $\hat{x} \in \mathscr{C}$ such that $\mathscr{F}=\mathscr{F}_{\hat{x}}$. Thus, (4.4) shows that $\bar{c}$ lies in $\operatorname{ri}(\operatorname{Normal}(\mathscr{C} ; \hat{x}))$, and so does $c$. It follows from Proposition 18 that $\max \{\langle c, x\rangle: \mathcal{A}(x)=b, x \in \mathbb{K}\}$ and its dual have a strictly complementary pair of optimal solutions.

One way to regard Theorem 22 is the following. Determining directly whether $\max \{\langle c, x\rangle: \mathcal{A}(x)=b, x \in \mathbb{K}\}$ and its dual have a pair of strictly complementary solutions individually for each $c \in \mathbb{E}^{*} \backslash[\operatorname{cone}(\mathscr{C})]^{\circ}$ involves studying a small portion of the boundary of infinitely many convex sets of the form $\left\{s \in \mathbb{K}^{*}: s \in \operatorname{Im}\left(\mathcal{A}^{*}\right)-c\right\}$, 
one for each objective vector $c$. Theorem 22 offers, as an alternative, determining the complete boundary structure of a single convex set, namely, $\mathscr{C}^{\circ}$.

In the same spirit as Proposition 1 the polar of the feasible region of a linear conic optimization problem may be described as follows (see, e.g., [20, Remark 2.2]):

Proposition 23. Let $\mathbb{K} \subseteq \mathbb{E}$ be a pointed closed convex cone with nonempty interior. Let $\mathcal{A}: \mathbb{E} \rightarrow \mathbb{Y}^{*}$ be a linear function, and let $b \in \mathbb{Y}^{*}$. Set $\mathscr{C}:=\{x \in \mathbb{K}: \mathcal{A}(x)=b\}$. Suppose that $\mathscr{C} \cap \operatorname{int}(\mathbb{K}) \neq \varnothing$. Let $\bar{x} \in \mathbb{E}$ such that $\mathcal{A}(\bar{x})=b$. Then

$$
\mathscr{C}^{\circ}=\left(\operatorname{Im}\left(\mathcal{A}^{*}\right) \cap\{\bar{x}\}^{\circ}\right)-\mathbb{K}^{*}
$$

Proof. By the Strong Duality Theorem, membership of $c \in \mathscr{C}^{\circ}$ is equivalent to the existence of $y \in \mathbb{Y}$ and $s \in \mathbb{K}^{*}$ such that $\mathcal{A}^{*}(y)-s=c$ and $\langle b, y\rangle \leq 1$. Note that $\langle b, y\rangle=\langle\mathcal{A}(\bar{x}), y\rangle=\left\langle\bar{x}, \mathcal{A}^{*}(y)\right\rangle$, so that $c \in \mathscr{C}^{\circ}$ if and only if $c \in\left(\operatorname{Im}\left(\mathcal{A}^{*}\right) \cap\{\bar{x}\}^{\circ}\right)-\mathbb{K}^{*}$.

Let us apply this in the context of MAxCut. Let $G=(V, E)$ be a graph, and let $w \in \mathbb{R}_{+}^{E}$. Let $\mathcal{L}_{G}(w):=\sum_{i j \in E} w_{i j}\left(e_{i}-e_{j}\right)\left(e_{i}-e_{j}\right)^{\top}$ denote the weighted Laplacian of $G$ with respect to $w$. The SDP relaxation for MAXCUT used by Goemans and Williamson [12] is

$$
\max \left\{\left\langle\frac{1}{4} \mathcal{L}_{G}(w), X\right\rangle: X \in \mathcal{E}_{V}\right\} .
$$

Note that $\mathcal{L}_{G}(w) \succeq 0$. Moreover, $\mathbb{S}_{+}^{V} \cap\left[\operatorname{cone}\left(\mathcal{E}_{V}\right)\right]^{\circ}=\{0\}$, since $I \in \mathcal{E}_{V}$. Thus, Theorem 22 and Proposition 23 yield a concrete approach to prove strict complementarity for all the "relevant" objective functions for the MaxCut SDP. Namely, it suffices to prove that $\mathcal{E}_{V}^{\circ}=\left(\operatorname{Im}(\operatorname{Diag}) \cap\{I\}^{\circ}\right)-\mathbb{S}_{+}^{V}$ is facially exposed.

\section{REFERENCES}

[1] A. Y. Alfakih. A remark on the faces of the cone of Euclidean distance matrices. Linear Algebra Appl., 414(1):266-270, 2006.

[2] F. Alizadeh, J.-P. A. Haeberly, and M. L. Overton. Complementarity and nondegeneracy in semidefinite programming. Math. Programming, 77(2, Ser. B):111-128, 1997. Semidefinite programming.

[3] A. Barvinok. A remark on the rank of positive semidefinite matrices subject to affine constraints. Discrete Comput. Geom., 25(1):23-31, 2001.

[4] A. I. Barvinok. Problems of distance geometry and convex properties of quadratic maps. Discrete Comput. Geom., 13(2):189-202, 1995.

[5] A. Bhardwaj, P. Rostalski, and R. Sanyal. Deciding polyhedrality of spectrahedra. http://arxiv.org/abs/1102.4367. December 2012. ArXiv e-prints, math.OC/1102.4367.

[6] J. Bolte, A. Daniilidis, and A. S. Lewis. Generic optimality conditions for semialgebraic convex programs. Math. Oper. Res., 36(1):55-70, 2011.

[7] J. P. R. Christensen and J. Vesterstrøm. A note on extreme positive definite matrices. Math. Ann., 244(1):65-68, 1979.

[8] C. B. Chua and L. Tunçel. Invariance and efficiency of convex representations. Math. Program., 111(1-2, Ser. B):113-140, 2008.

[9] M. K. de Carli Silva. Geometric Ramifications of the Lovász Theta Function and Their Interplay with Duality. PhD thesis, Department of Combinatorics and Optimization, University of Waterloo, 2013.

[10] C. De Simone. The cut polytope and the Boolean quadric polytope. Discrete Math., 79(1):71-75, 1989/90.

[11] D. Drusvyatskiy and A. S. Lewis. Generic nondegeneracy in convex optimization. Proc. Amer. Math. Soc., 139(7):2519$2527,2011$.

[12] M. X. Goemans and D. P. Williamson. Improved approximation algorithms for maximum cut and satisfiability problems using semidefinite programming. J. Assoc. Comput. Mach., 42(6):1115-1145, 1995.

[13] S. J. Gortler and D. P. Thurston. Characterizing the universal rigidity of generic frameworks. ArXiv e-prints, December 2010.

[14] M. Grötschel, L. Lovász, and A. Schrijver. Polynomial algorithms for perfect graphs. In Topics on perfect graphs, volume 88 of North-Holland Math. Stud., pages 325-356. North-Holland, Amsterdam, 1984.

[15] M. Grötschel, L. Lovász, and A. Schrijver. Relaxations of vertex packing. J. Combin. Theory Ser. B, 40(3):330-343, 1986.

[16] N. Gvozdenović and M. Laurent. The operator $\Psi$ for the chromatic number of a graph. SIAM J. Optim., 19(2):572-591, 2008.

[17] J. Kleinberg and M. X. Goemans. The Lovász theta function and a semidefinite programming relaxation of vertex cover. SIAM J. Discrete Math., 11(2):196-204 (electronic), 1998.

[18] D. E. Knuth. The sandwich theorem. Electron. J. Combin., 1:Article 1, approx. 48 pp. (electronic), 1994.

[19] D. G. Larman. On a conjecture of Klee and Martin for convex bodies. Proc. London Math. Soc. (3), 23:668-682, 1971.

[20] M. Laurent and S. Poljak. On a positive semidefinite relaxation of the cut polytope. Linear Algebra Appl., 223/224:439-461, 1995. Special issue honoring Miroslav Fiedler and Vlastimil Pták.

[21] M. Laurent and S. Poljak. On the facial structure of the set of correlation matrices. SIAM J. Matrix Anal. Appl., 17(3):530$547,1996$. 
[22] M. Laurent, S. Poljak, and F. Rendl. Connections between semidefinite relaxations of the max-cut and stable set problems. Math. Programming, 77(2, Ser. B):225-246, 1997. Semidefinite programming.

[23] C.-K. Li and B. S. Tam. A note on extreme correlation matrices. SIAM J. Matrix Anal. Appl., 15(3):903-908, 1994.

[24] R. Loewy. Extreme points of a convex subset of the cone of positive semidefinite matrices. Math. Ann., 253(3):227-232, 1980.

[25] L. Lovász. On the Shannon capacity of a graph. IEEE Trans. Inform. Theory, 25(1):1-7, 1979.

[26] L. Lovász and A. Schrijver. Cones of matrices and set-functions and 0-1 optimization. SIAM J. Optim., 1(2):166-190, 1991.

[27] R. J. McEliece, E. R. Rodemich, and H. C. Rumsey, Jr. The Lovász bound and some generalizations. J. Combin. Inform. System Sci., 3(3):134-152, 1978.

[28] J. Nie, K. Ranestad, and B. Sturmfels. The algebraic degree of semidefinite programming. Math. Program., 122(2, Ser. A):379-405, 2010.

[29] G. Pataki. Cone-LP's and semidefinite programs: geometry and a simplex-type method. In Integer programming and combinatorial optimization (Vancouver, BC, 1996), volume 1084 of Lecture Notes in Comput. Sci., pages 162-174. Springer, Berlin, 1996.

[30] G. Pataki. On the rank of extreme matrices in semidefinite programs and the multiplicity of optimal eigenvalues. Math. Oper. Res., 23(2):339-358, 1998.

[31] G. Pataki and L. Tunçel. On the generic properties of convex optimization problems in conic form. Math. Program., 89(3, Ser. A):449-457, 2001.

[32] R. T. Rockafellar. Convex analysis. Princeton Landmarks in Mathematics. Princeton University Press, Princeton, NJ, 1997. Reprint of the 1970 original, Princeton Paperbacks.

[33] J. Saunderson, V. Chandrasekaran, P. A. Parrilo, and A. S. Willsky. Diagonal and low-rank matrix decompositions, correlation matrices, and ellipsoid fitting. ArXiv e-prints, April 2012.

[34] A. Schrijver. A comparison of the Delsarte and Lovász bounds. IEEE Trans. Inform. Theory, 25(4):425-429, 1979.

[35] A. Schrijver. Combinatorial optimization. Polyhedra and efficiency. Vol. B, volume 24 of Algorithms and Combinatorics. Springer-Verlag, Berlin, 2003. Matroids, trees, stable sets, Chapters 39-69.

[36] F. B. Shepherd. The theta body and imperfection. In Perfect graphs, Wiley-Intersci. Ser. Discrete Math. Optim., pages 261-291. Wiley, Chichester, 2001.

[37] M. Szegedy. A note on the theta number of Lovász and the generalized Delsarte bound. In Proceedings of the 35th Annual IEEE Symposium on Foundations of Computer Science, 1994.

[38] H. Wolkowicz, R. Saigal, and L. Vandenberghe, editors. Handbook of semidefinite programming. International Series in Operations Research \& Management Science, 27. Kluwer Academic Publishers, Boston, MA, 2000. Theory, algorithms, and applications.

\section{Appendix A. Proof of Proposition 20}

Proof. We may assume that $\mathscr{C} \neq \varnothing$, so that $\gamma(\cdot \mid \mathscr{C})$ is proper. Thus, we have to show that $\gamma(\cdot \mid \mathscr{C})$ is lower semi-continuous, i.e., for each $\alpha \in \mathbb{R}$, the sub-level set $S_{\alpha}:=\{x \in \mathbb{E}: \gamma(x \mid \mathscr{C}) \leq \alpha\}$ is closed. This is clearly the case for $\alpha<0$. For $\alpha \geq 0$, we shall the fact that

$$
\text { there exists } M>0 \text { such that } \gamma(x \mid \mathscr{C}) \geq\|x\| / M \text { for all } x \in \mathbb{E} \text {. }
$$

Indeed, set $M:=\max \{\|x\|: x \in \mathscr{C}\}+1$. Then, if $\lambda \geq 0$ is such that $x \in \lambda \mathscr{C}$, we have $\|x\| \leq \lambda M$. This proves A.1).

Note that (A.1) implies that $S_{0}=\{0\}$. Next, let $\alpha>0$. Let $\left(x_{n}\right)_{n \in \mathbb{N}}$ be a sequence in $S_{\alpha}$ converging to $x$. If $x \neq 0$, then $x \in S_{0} \subseteq S_{\alpha}$, so assume that $x \neq 0$. By taking some tail of the sequence, we may assume that $\left\|x_{n}\right\| \geq \frac{1}{2}\|x\|$ for each $n$. Set $\gamma_{n}:=\gamma\left(x_{n} \mid \mathscr{C}\right)$, so that $\frac{\|x\|}{2 M} \leq \frac{\left\|x_{n}\right\|}{M} \leq \gamma_{n} \leq \alpha$ for each $n$. Refine the sequence so that $\gamma_{n} \rightarrow \gamma$ for some $\gamma \in \mathbb{R}$ with $\frac{\|x\|}{2 M} \leq \gamma \leq \alpha$. For each $n$, there exists $0 \leq \varepsilon_{n} \leq 1 / n$ such that $x_{n} \in\left(\gamma_{n}+\varepsilon_{n}\right) \mathscr{C}$, whence

$$
\frac{1}{\gamma_{n}+\varepsilon_{n}} x_{n} \in \mathscr{C} \quad \forall n \in \mathbb{N}
$$

By taking $n \rightarrow \infty$, we find that $\frac{1}{\gamma} x \in \mathscr{C}$, so that $\gamma(x \mid \mathscr{C}) \leq \gamma \leq \alpha$ and $x \in S_{\alpha}$.

\section{Appendix B. Proof of Theorem 21}

Proof. It is easy to check that

$$
\mathscr{F}_{\bar{x}}=\left\{y \in \mathscr{C}^{\circ}:\langle y, \bar{x}\rangle=1\right\} \quad \forall \bar{x} \in \mathscr{C} .
$$

Note that every set of the form $\left\{y \in \mathscr{C}^{\circ}:\langle y, \bar{x}\rangle=1\right\}$ for some $\bar{x} \in \mathscr{C}$ is an exposed face of $\mathscr{C}$. Furthermore $0 \in \mathscr{C}^{\circ}$ shows that $\mathscr{F}_{\bar{x}}$ is a proper subset of $\mathscr{C}^{\circ}$.

Next let $\mathscr{F}$ be a nonempty exposed face of $\mathscr{C}^{\circ}$ other than $\mathscr{C}^{\circ}$ itself, so that

$$
\mathscr{F}=\left\{y \in \mathscr{C}^{\circ}:\langle y, \bar{z}\rangle=\delta^{*}\left(\bar{z} \mid \mathscr{C}^{\circ}\right)\right\}
$$


for some $\bar{z} \in \mathbb{E}$ such that $\delta^{*}\left(\bar{z} \mid \mathscr{C}^{\circ}\right)<\infty$. Then $\mathscr{F} \neq \mathscr{C}^{\circ}$ shows that $\bar{z} \neq 0$. By Proposition 20 we have $\gamma(x \mid \mathscr{C})=\delta^{*}\left(x \mid \mathscr{C}^{\circ}\right)$ for every $x \in \mathbb{E}$; see, e.g., [32, p. 125]. Thus, $0<\gamma(\bar{z} \mid \mathscr{C})=\delta^{*}\left(\bar{z} \mid \mathscr{C}^{\circ}\right)<\infty$. Set $\bar{x}:=\bar{z} / \gamma(\bar{z} \mid \mathscr{C})$, so that $\gamma(\bar{x} \mid \mathscr{C})=1$. Then

$$
\mathscr{F}=\left\{y \in \mathscr{C}^{\circ}:\langle y, \bar{x}\rangle=1\right\}=\mathscr{F}_{\bar{x}}
$$

by (B.1). This completes the precise description of all the nonempty exposed faces of $\mathscr{C}^{\circ}$.

Finally, let $\bar{x} \in \mathscr{C}$ such that $\mathscr{F}_{\bar{x}} \neq \varnothing$. To prove (4.4), it suffices to prove that $\operatorname{ri}(\operatorname{Normal}(\mathscr{C} ; \bar{x}))$ meets $\left\{y \in \mathbb{E}^{*}:\langle y, \bar{x}\rangle=1\right\}$; see, e.g., [32, Theorem 6.5]. Suppose not. Then there is a hyperplane separating $\operatorname{Normal}(\mathscr{C} ; \bar{x})$ and the second set, i.e., there exists a nonzero $h \in \mathbb{E}$ and $\alpha \in \mathbb{R}$ such that

$$
\begin{gathered}
\operatorname{Normal}(\mathscr{C} ; \bar{x}) \subseteq\left\{y \in \mathbb{E}^{*}:\langle y, h\rangle \leq \alpha\right\}, \\
\left\{y \in \mathbb{E}^{*}:\langle y, \bar{x}\rangle=1\right\} \subseteq\left\{y \in \mathbb{E}^{*}:\langle y, h\rangle \geq \alpha\right\} .
\end{gathered}
$$

Note that $0 \in \operatorname{Normal}(\mathscr{C} ; \bar{x})$ shows that $\alpha \geq 0$, and that $h=\lambda \bar{x}$ for some $\lambda>0$. Positive homogeneity now shows that $\langle y, h\rangle \leq 0$ for all $y \in \operatorname{Normal}(\mathscr{C} ; \bar{x})$, whence $\mathscr{F}_{\bar{x}}=\varnothing$, a contradiction. 